\title{
ADDITIVE FUNCTIONALS OF MARKOV PROCESSES IN DUALITY
}

\author{
BY \\ R. M. BLUMENTHAL AND R. K. GETOOR(1)
}

1. Introduction. The present paper may be regarded as a continuation of [3]. In [3] we showed that if $X$ is a Hunt process (i.e., satisfies Hunt's hypothesis (A)) then there is a one-to-one correspondence between strictly increasing continuous additive functionals $A$ of $X$ (subject to certain finiteness requirements) and Hunt processes $Y$ with the same hitting distributions as $X$. This correspondence is given by the relationship that $Y$ is stochastically equivalent to $t \rightarrow X[\tau(t)]$ where $\tau$ is the functional inverse to $A$. In this paper we investigate this relationship under the additional assumption that $X$ satisfies Hunt's hypothesis (F) as well as (A).

We begin by setting up a one-to-one correspondence between the appropriate class of additive functionals and a certain class of measures (called smooth) on the state space. This is described in $\$ \S 4-6$ ( $\$ \S 2$ and 3 are of a preliminary nature). This correspondence was set up by McKean and Tanaka [7] in the case that $X$ is Brownian motion; and our presentation is similar to theirs. $§ 7$ then relates this correspondence to processes $Y$ with the same hitting distributions as $X$. Since $X$ satisfies hypothesis $(\mathrm{F})$ there exists another Hunt process $\not{X}$ called the dual of $X$ (see $\$ 2$ for definitions). It is natural to ask if given $Y$ with the same hitting distributions as $X$, then does there exist $\hat{Y}$ with the same hitting distributions as $X$ and which is dual in some sense to $Y$. $\S \S 8$ and 9 are devoted to giving an essentially affirmative answer to this question.

2. Preliminaries. In the present paper we will adopt the terminology and notation of P. A. Meyer [8] with only minor changes. In order to fix the notation we review briefly the basic definitions. The state space $E$ is a locally compact Hausdorff space with a countable base for its topology. (Meyer uses $X$ instead of $E$.) Let $\Delta$ be a point adjoined to $E$ as the point at infinity if $E$ is noncompact and as an isolated point if $E$ is compact. We write $\bar{E}=E \cup\{\Delta\}$. Let $\mathscr{B}(\overline{\mathscr{B}})$ be the topological Borel field of $E(\bar{E})$ and let $\mathscr{A}(\overline{\mathscr{A}})$ be the $\sigma$-algebra of universally measurable subsets of $E(\bar{E})$ that is, $A$ is in $\mathscr{A}(\overline{\mathscr{A}})$ if it is in the completion of $\mathscr{B}(\overline{\mathscr{B}})$ with respect to all finite measures $\mu$ on $\mathscr{B}(\overline{\mathscr{B}})$. Let $\Omega^{*}$ denote the space of all maps $\omega^{*}$ from $[0, \infty]$ into $E$ such that $\omega^{*}$ is right continuous and has left-hand

Received by the editors March 18, 1963.

(1) This research was supported in part by the National Science Foundation, Grant No. G-21205. 
limits on $[0, \infty), \omega^{*}(\infty)=\Delta$, and if $\omega^{*}\left(t_{0}\right)=\Delta$ then $\omega^{*}(t)=\Delta$ for all $t \geqq t_{0}$. As usual, $X_{t}\left(\omega^{*}\right)=X\left(t, \omega^{*}\right)=\omega^{*}(t)$ denotes the $t$ th coordinate function and $\mathscr{F}_{s}^{0}\left(\mathscr{F}^{0}\right)$ denotes the $\sigma$-algebra of subsets of $\Omega^{*}$ generated by sets of the form $X_{t}^{-1}(A)$ where $A$ is in $\mathscr{B}$ and $t \leqq s(t<\infty)$. The shift operators $\theta_{t}$ are defined on $\Omega^{*}$ by $\theta_{t} \omega^{*}(s)=\omega^{*}(t+s)$. We assume that for each $x$ in $\bar{E}$ we are given a probability measure $P^{x}$ on $\mathscr{F}^{0}$ satisfying:

(i) $x \rightarrow P^{x}(\Lambda)$ is $\overline{\mathscr{B}}$ measurable for each $\Lambda \in \mathscr{F}^{0}$; and

(ii) $P^{x}[X(0)=x]=1$ for all $x$ in $\bar{E}$.

Note that (ii) implies that $P^{\Delta}$ is unit mass at the function $\omega^{*}$ which is identically equal to $\Delta$.

For each finite measure $\mu$ on $\overline{\mathscr{B}}$ we define $P^{\mu}(\Lambda)=\int P^{x}(\Lambda) \mu(d x)$ for $\Lambda$ in $\mathscr{F}^{0}$ and we then define $\mathscr{F}^{*}\left(\mathscr{F}_{t}^{*}\right)$ to be the intersection over all such $\mu$ of the $P^{\mu}$ completions of $\mathscr{F}^{0}\left(\mathscr{F}_{t}^{0}\right)$. Now let $\left(\Omega_{0}, \mathscr{G}, P\right)$ be any sufficiently large probability space and set $\Omega=\Omega^{*} \times \Omega_{0}, \mathscr{F}=\mathscr{F}^{*} \times \mathscr{G}\left(\mathscr{F}_{t}=\mathscr{F}_{t}^{*} \times \mathscr{G}\right)$; and, at the risk of momentary confusion, redefine $\mathscr{F}_{t}^{*}=\mathscr{F}_{t}^{*} \times\left\{\varnothing, \Omega_{0}\right\}$ and $P^{\mu}=P^{\mu} \times P$. If $\omega=\left(\omega^{*}, \omega_{0}\right)$ is a typical point of $\Omega$ define the shift operator $\theta_{t}$ by $\theta_{t} \omega=\theta_{t}\left(\omega^{*}, \omega_{0}\right)=\left(\theta_{t} \omega^{*}, \omega_{0}\right)$ and also set $X_{t}(\omega)=X_{t}\left(\omega^{*}\right)$. It follows that $x \rightarrow P^{x}(\Lambda)$ is $\mathscr{A}^{-}$measurable for each $\Lambda$ in $\mathscr{F}$ and that $(t, \omega) \rightarrow X_{t}(\omega)$ is jointly measurable with respect to $\mathscr{T} \times \mathscr{F}$ and $\overline{\mathscr{B}}$. Here $\mathscr{T}$ denotes the Borel sets of $[0, \infty)$. A function $T: \Omega \rightarrow[0, \infty]$ is called a stopping time if $\{T<t\}$ is in $\mathscr{F}_{t}$ for each $t>0$, and for such a stopping time $\mathscr{F}_{T}$ denotes the $\sigma$-algebra of all sets $\Lambda$ in $\mathscr{F}$ such that $\Lambda \cap\{T<t\} \in \mathscr{F}_{\text {t }}$ for all $t>0$. We assume (in addition to (i) and (ii) above):

(iii) For any increasing sequence $\left\{T_{n}\right\}$ of stopping times with limit $T$ we have $X\left(T_{n}\right) \rightarrow X(T)$ almost surely on $\{T<\infty\}$. Here and henceforth almost surely (abbreviated a.s.) means almost surely with respect to each $P^{x}$.

(iv) Strong Markov Property. For each stopping time $T$, bounded measurable function $F$ on $\Omega$, and $\Lambda$ in $\mathscr{F}_{T}$ we have

$$
E^{x}\left\{F\left(\theta_{T} \omega\right) ; \Lambda\right\}=E^{x}\left\{E^{X(T)}(F) ; \Lambda\right\}
$$

for all $x$. Here $E^{x}(F ; \Lambda)=\int_{\Lambda} F d P^{x}$.

Using the terminology introduced in [3] a process $X=\left(E, P^{x}\right)$ satisfying (i)-(iv) is called a Hunt process. Such processes are exactly those satisfying Hunt's hypothesis (A) $[5, \mathrm{I}]$. We will use the standard properties of such processes without mention. See [4], [5], or [10]; also the required properties are outlined in [3] and [8]. As usual $P_{t}(x, d y)=P^{x}\left(X_{t} \in d y\right)$ is the transition function of $X$, and we adopt the convention that any function $f$ defined on $E$ is extended to $\bar{E}$ by setting $f(\Delta)=0$, unless explicitly stated otherwise. We let $\sigma=\inf \left\{t>0: X_{t}=\Delta\right\}$ be the lifetime of the process. (Meyer [8] uses $S$ in place of $\sigma$.)

If $A$ is an analytic subset of $\bar{E}$ we let $T_{A}=\inf \{t>0: X(t) \in A\}$ be the first hitting time of $A$, which is known [5] to be a stopping time. Also $P^{x}\left(T_{A}=0\right)$ is either zero or one, and in the latter case $x$ is said to be regular for $A$. More generally if $A$ is any set, $x$ is not regular (irregular) for $A$ if there exists a Borel set $B$ con- 
taining $A$ such that $P^{x}\left(T_{B}>0\right)=1$. A set $A$ is said to be finely open if each point in $A$ is irregular for $\bar{E}-A$. The collection of all finely open sets is a topology on $E$ called the fine topology induced by $X$. See [10] for a discussion of the fine topology. In particular it is not difficult to see that the finely open Borel sets form a base for the fine topology.

Throughout the remainder of this paper we assume that $X=\left(E, P^{x}\right)$ is a Hunt process which, in addition, satisfies Hunt's hypothesis (F) [5, III, p. 154]. We will now recall some of the consequences of $(F)$ that we will use in the sequel. There exists on $E$ a positive measure $\xi$ (we will write $d x$ for $\xi(d x)$ ) finite on compacts and positive on nonvoid open sets. In addition there exists another Hunt process $\hat{X}=\left(E, \hat{P}^{x}\right)$ which will be referred to as the dual process. The basic measure $\xi$ is excessive with respect to both $X$ and $\hat{X}$, that is, for $A$ in $\mathscr{A}$

$$
\int d x P_{t}(x, A) \leqq \xi(A) ; \quad \int \hat{P}_{t}(A, x) d x \leqq \xi(A)
$$

for each $t>0$. Here $\hat{P}_{t}(d y, x)$ is the transition function of $\hat{X}$. Note the unusual order of the symbols. We will follow the terminology of Meyer [8, part II, §6] and designate quantities defined relative to $\hat{X}$ by the prefix "co." Thus we will speak of coexcessive functions, the cofine topology, etc. This differs from Hunt's terminology in which the prefixes "right" and "left" designate quantities defined relative to $X$, and $\hat{X}$ respectively.

We now list the fundamental properties that we will use:

(a) The space $C_{0}(E)$ is invariant under the semi-groups

$$
P_{t} f(x)=\int P_{t}(x, d y) f(y) ; \quad \hat{P}_{t} f(x)=\int f(y) \hat{P}_{t}(d y, x),
$$

where $C_{0}(E)$ denotes the continuous functions on $E$ that vanish at infinity.

(b) For each $\lambda \geqq 0$ there exists a function $U^{\lambda}(x, y)$ on $E \times E$ such that the potential kernels for $X$ and $\hat{X}$ are given by

$$
U^{\lambda}(x, d y)=U^{\lambda}(x, y) d y ; \quad \hat{U}^{\lambda}(d x, y)=d x U^{\lambda}(x, y)
$$

where as usual

$$
U^{\lambda}(x, A)=\int_{0}^{\infty} e^{-\lambda t} P_{t}(x, A) d t ; \quad \hat{U}^{\lambda}(A, x)=\int_{0}^{\infty} e^{-\lambda t} \hat{P}_{t}(A, x) d t .
$$

The functions $U^{\lambda}(x, y)$ are separately lower semi-continuous in $x$ and $y$ and if $f$ is a bounded $\mathscr{A}$ measurable function vanishing outside a compact subset of $E$ then for $\lambda>0$ the functions

$$
x \rightarrow \int U^{\lambda}(x, y) f(y) d y, \quad y \rightarrow \int f(x) U^{\lambda}(x, y) d x
$$

belong to $C_{0}(E)$. Given a measure $\mu$ we can then define the potential $U^{\lambda} \mu$ 
(copotential $\left.\mu U^{\lambda}\right)$ of $\mu$, which is a $\lambda$-excessive ( $\lambda$-coexcessive) function. ( $U=U^{0}$ may be identically infinite.)

(c) Excessive (coexcessive) functions are lower semi-continuous.

(d) If $A$ is an analytic subset of $\bar{E}$ then $T_{A}$, the first hitting time of $A$, is a stopping time for both $X$ and $\hat{X}$. Let $S^{\lambda}$ denote a non-negative random variable that is independent of both $X$ and $\hat{X}$, and has an exponential distribution with parameter $\lambda>0$. If we define

$$
P_{A}^{\lambda}(x, D)=P^{x}\left[X\left(T_{A}\right) \in D ; \quad T_{A}<S^{\lambda}\right]=E^{x}\left[e^{-\lambda T_{A}} ; \quad X\left(T_{A}\right) \in D\right]
$$

and $\hat{P}_{A}^{\lambda}(D, x)$ in a similar fashion relative to $\hat{X}$, then the following identity is the basic consequence of $(F)$ :

$$
P_{A}^{\lambda} U^{\lambda}(x, y)=\int P_{A}^{\lambda}(x, d z) U^{\lambda}(z, y)=\int U^{\lambda}(x, z) \hat{P}_{A}^{\lambda}(d z, y)=U^{\lambda} \hat{P}_{A}^{\lambda}(x, y) .
$$

See Hunt $\left[5\right.$, III, p. 168]. Note that Hunt writes $H_{A}^{\lambda}$ in place of $P_{A}^{\lambda}$.

(e) If $\phi$ is an excessive (coexcessive) function, then the following statements are equivalent [5, III, p. 167]:

(i) $\phi$ is finite on a set dense in $E$.

(ii) $\phi$ is finite a.e. on $E$; almost everywhere refers to the basic measure $\xi$ unless explicitly stated otherwise.

(iii) $\phi$ is locally integrable with respect to $\xi$.

(f) The basic measure $\xi$ is positive on nonvoid finely (cofinely) open Borel sets and hence if $A$ in $\mathscr{A}$ has measure zero, then $E-A$ is both finely and cofinely dense in $E$. Thus if two excessive functions agree a.e. they are identical.

The $\sigma$-algebras $\hat{\mathscr{F}}_{t}^{*}, \hat{\mathscr{F}}_{t}$, etc. are defined relative to $\hat{X}$ as $\mathscr{F}_{t}^{*}, \mathscr{F}_{t}$, etc. are defined relative to $X$. Since in general $\mathscr{F}_{t}^{*}$ and $\hat{\mathscr{F}}_{t}^{*}$ need not be the same, the fact that $T$ is a stopping time for $X$ does not imply that $T$ is a stopping time for $\hat{X}$. Of course, if $A$ is an analytic set then $T_{A}$ is a stopping time for both $X$ and $\hat{X}$, and a similar statement holds for limits of such stopping times.

A set $A \subset E$ is polar (thin) if $A$ is contained in a Borel set $B$ such that $P^{x}\left(T_{B}=\infty\right)=1\left(P^{x}\left(T_{B}>0\right)=1\right)$ for all $x$ in $E$. A set is semi-polar if it is contained in a countable union of thin sets. These definitions differ slightly from those given by Meyer [8, p. 159] but are equivalent to his under hypothesis $(F)$ (or more generally under Meyer's hypothesis (L); see [8, p. 160 and p. 163]). In [5] Hunt uses the terminology negligible instead of polar. Meyer [8, p. 214] has shown that a set is polar (semi-polar) if and only if it is copolar (cosemi-polar) so that these concepts coincide for the two processes $X$ and $\hat{X}$. The following result will be useful in the sequel.

THEOREM 2.1. Let $A$ be a Borel subset of $E$ with compact closure contained in $E$ and let $B(\hat{B})$ be the set of points which are not regular (coregular) for $A^{c}=\hat{E}-A$. Then $B-\hat{B}$ and $\hat{B}-B$ are semi-polar. 
Proof. Let $F=B-\hat{B}$ and note that every point that is either in $F$ or coregular for $F$ is coregular for $A^{c}$. Let $\lambda>0$ and define $\Phi_{F}^{\lambda}(x)=E^{x}\left[e^{-\lambda T_{F}}\right]$. Arguing as in [5, III, Proposition 21.1] we have

$$
\Phi_{F}^{\lambda}=U^{\lambda} \Pi_{F}^{\lambda}=U^{\lambda} \hat{P}_{A^{c}}^{\lambda} \Pi_{F}^{\lambda}=P_{A^{c}}^{\lambda} \Phi_{F}^{\lambda}
$$

where $\Pi_{F}^{\lambda}$ is the natural $\lambda$-capacitary measure of $F$, which is concentrated on $F$ union the points coregular for $F$. Since $P_{A^{c}}^{\lambda}(x, E)<1$ for $x$ in $F$ and $\Phi_{F}^{\lambda} \leqq 1$, it follows that $\Phi_{F}^{\lambda}<1$ on $F$. Define $F_{n}=\left\{x \in F: \Phi_{F}^{\lambda}(x) \leqq 1-1 / n\right\}$ so that $F=\bigcup F_{n}$. We claim that each $F_{n}$ is thin. If $y$ is not regular for $F$ it certainly is not regular for $F_{n}$. If $y$ is regular for $F$ then $\Phi_{F}^{\lambda}(y)=1$ and so $y$ is not regular for any $F_{n}$. Hence each $F_{n}$ is thin and consequently $F$ is semi-polar. Dually $\hat{B}-B$ is cosemi-polar and hence semi-polar.

An argument similar to that above shows that if $A$ is analytic then the set of points in $A$ which are not regular for $A$ is semi-polar.

A non-negative $\mathscr{A}$ measurable function $\phi$ is called super mean valued if $P_{t} \phi \leqq \phi$ for all $t>0$. It is easy to see that then $P_{t} \phi$ increases as $t$ decreases to zero and the limit $\phi^{*}$ is an excessive function dominated by $\phi$. One calls $\phi^{*}$ the (excessive) regularization of $\phi$. The following result is due to Doob and will be needed in the sequel; for a proof see [10, Chapter 9].

THEOREM 2.2. Let $\left\{\phi_{n}\right\}$ be a decreasing sequence of excessive functions with limit $\phi$. Then $\phi$ is super mean valued and it differs from its regularization $\phi^{*}$ on at most a semi-polar set.

3. Exit sets. We will assume throughout the remainder of this paper that $X$ is a Hunt process satisfying hypothesis (F) on $E$ with dual process $\hat{X}$. We will also assume that neither $X$ nor $\hat{X}$ have any traps in $E$ ( $x$ is a trap for $X$ if $\left.P^{x}\left(T_{\{x\}^{c}}=\infty\right)=1\right)$. An open set $G$ with compact closure $G$ in $E$ is said to be an exit set if $E^{x}\left(T_{\bar{G}}\right)$ and $\hat{E}^{x}\left(T_{\bar{G}}\right)$ are both bounded in $x$. For typographical convenience we will let $G^{\prime}$ denote $\bar{G}^{c}=\bar{E}-\bar{G}$ whenever $G$ is an exit set. The proof of the following lemma is elementary and hence is omitted. See [6, p. 640].

Lemma 3.1. If $A$ is a Borel set and if there exist $t>0$ and $\beta<1$ such th a $P^{x}\left(T_{A^{c}}>t\right)<\beta$ for all $x$, then $E^{x}\left(T_{A^{c}}\right)$ is bounded.

The next theorem is the main result of the present section. Let $\rho$ be a fixed metric on $E$ that is compatible with the given topology.

THEOREM 3.2. There exist a countable collection $\left\{G_{j} ; j \geqq 1\right\}$ of exit sets that covers $E$ and a Borel measurable function $N$ from $E$ to the positive integers such that:

(i) $x \in G_{N(x)}$ for all $x$.

(ii) If $K$ is compact then $\rho\left(x, G_{N(x)}^{c}\right)$ is bounded away from zero on $K$.

Proof. Let $\left\{N_{i}\right\}$ be a countable family of open sets with compact closures forming a base for the topology of $E$. Define 


$$
\begin{aligned}
& u_{i}(x)=\int_{0}^{\infty} e^{-t} P_{t}\left(x, \bar{N}_{i}\right) d t, \\
& \hat{u}_{i}(x)=\int_{0}^{\infty} e^{-t} \hat{P}_{t}\left(\bar{N}_{i}, x\right) d t
\end{aligned}
$$

and set $W_{i j}=N_{i} \cap\left\{u_{i}<1-1 / j\right\} \cap\left\{\hat{u}_{i}<1-1 / j\right\}$. Since $u_{i}$ and $\hat{u}_{i}$ are continuous (property (b) of hypothesis (F)) each $W_{i j}$ is open and $E=\bigcup W_{i j}$ since there are no traps in $E$ for either $X$ or $\hat{X}$. Moreover, $W_{i j} \subset \bar{N}_{i} \cap\left\{u_{i} \leqq 1-1 / j\right\} \cap\left\{\hat{u}_{i} \leqq 1-1 / j\right\}$ and it then follows easily from Lemma 3.1 that each nonvoid $W_{i j}$ is an exit set. Let $\left\{G_{n}\right\}$ be an enumeration of the distinct nonvoid $W_{i j}$. Clearly $E=\bigcup G_{n}$.

If we define

$$
H_{i j}=\left\{y: \rho\left(y, G_{i}^{c}\right)>\frac{1}{j}\right\}
$$

then $I_{i j}$ is open and $\bigcup H_{i j}=E$. Let $\left\{V_{n}\right\}$ be an enumeration of the distinct nonvoid $H_{i j}$, and define as follows:

$$
\begin{aligned}
& M(x)=\inf \left\{j: x \in V_{j}\right\} \\
& m(k)=\text { that } i \text { such that } V_{k}=H_{i j} \\
& N(x)=m(M(x))
\end{aligned}
$$

It is evident that $x \in G_{N(x)}$ for each $x$ in $E$, and that $x \rightarrow N(x)$ is Borel measurable. Let $K$ be a compact subset of $E$ and choose $j_{0}$ so that $V_{1}, \cdots, V_{j_{0}}$ cover $K$. Let $k_{0}$ be largest value of $k$ such that $H_{i k}$ appears in the list $V_{1}, \cdots, V_{j_{0}}$. Thus any $x$ in $K$ is in $H_{N(x) k}$ for some $k \leqq k_{0}$, and so $\rho\left(x, G_{N(x)}^{c}\right) \geqq k_{0}^{-1}$ for all $x$ in $K$. This completes the proof of Theorem 3.2.

The next result will also prove useful in the sequel.

THEOREM 3.3. Let $K$ be a compact subset of $E$ and suppose that $P^{x}\left(T_{K^{c}}<\infty\right)>0$ and $\hat{P}^{x}\left(T_{K^{c}}<\infty\right)>0$ for each $x$ in $K$. Then there exists an exit set $G$ with $K \subset G$.

Proof. The function $f(x)=\int_{0}^{\infty} e^{-t} P_{t}(x, K) d t$ is continuous and strictly less than 1 on $K$ according to our hypothesis. Let $H$ be a neighborhood of $K$ with compact closure on which $f$ is bounded away from 1 . Let $\left\{G_{n}\right\}$ be a decreasing sequence of open sets contained in $H$ with $\bar{G}_{n+1} \subset G_{n}$ and $\bigcap G_{n}=K$. If $f_{n}(x)=\int_{0}^{\infty} e^{-t} P_{t}\left(x, G_{n}\right) d t$, then the $f_{n}$ are continuous and decrease to $f$. Hence they decrease to $f$ uniformly in $H$. If $n$ is large enough that $f_{n}$ is bounded away from 1 on $H$, then Lemma 3.1 implies that $E^{x}\left(T_{G_{n+1}^{\prime}}\right)$ is bounded in $x$. In the same way we can find a neighborhood $J$ of $K$ with compact closure such that $\hat{E}^{x}\left(T_{J}\right)$ is bounded. Clearly $G=G_{n+1} \cap J$ has the desired properties. 
4. Continuous additive functionals: local theory. We assume that $X$ and $\hat{X}$ are as in $\S 3$. The family of random variables $A=\{A(t) ; t \geqq 0\}$ is said to be a continuous additive functional of $X$ if

(i) $A(0)=0,0 \leqq A(t) \leqq \infty$ for all $t \geqq 0, t \rightarrow A(t)$ is increasing and continuous (relative to the topology of the extended real line) on $[0, \infty]$, and $A(t)=A(\sigma)$ for all $t \geqq \sigma$; all of these statements holding almost surely.

(ii) $A(t)$ is $\mathscr{F}_{t}{ }^{*}$ measurable for each $t \geqq 0$.

(iii) For each fixed $t$ and $s$ we have $A(t+s, \omega)=A(t, \omega)+A\left(s, \theta_{t} \omega\right)$ almost surely.

Meyer [8] has shown that a continuous additive functional (hereafter abbreviated c.a.f.) has the following strong Markov property: If $T$ is a stopping time and $H$ a random variable such that $H \geqq 0$ a.s., then

$$
A[H(\omega)+T(\omega), \omega]=A[T(\omega), \omega]+A\left[H(\omega), \theta_{T} \omega\right] \text { a.s. }
$$

In general when writing such statements we will omit the $\omega$ 's.

Let $G$ be a fixed exit set and let $T=T_{G^{\prime}}$; then $(X, T)$ denotes the processes $X$ terminated at time $T$. Let $B$ and $\hat{B}$ be the state spaces for $(X, T)$ and $(\hat{X}, T)$, i.e., $B(\hat{B})$ consists of all points not regular (coregular) for $G^{\prime}$. Clearly $B(\hat{B})$ is a finely (cofinely) open Borel set and, according to Theorem $2.1, B-\hat{B}$ and $\hat{B}-B$ are semi-polar. Let $V^{\lambda}(x, y), \lambda \geqq 0$, be the potential kernels for $(X, T)$ and $(\hat{X}, T)$. See Hunt [5, III, §21] for the existence and properties of $V^{\lambda}$. Note that the fact that $G$ is an exit set implies that hypothesis $\left(\mathrm{G}^{*}\right)$ of Hunt [5, III, $\S 21]$ holds. If $\phi$ is excessive relative to $(X, T)$ then $\phi$ is Borel measurable. (See the argument above Proposition 21.2 of [5, III].) If $D$ is an analytic set, we write (following Hunt)

$$
\begin{aligned}
K_{D}^{\lambda}(x, d y) & =P^{x}\left[X\left(T_{D}\right) \in d y ; T_{D}<\min \left(T, S^{\lambda}\right)\right] \\
& =E^{x}\left[e^{-\lambda T_{D}} ; X\left(T_{D}\right) \in d y, T_{D}<T\right],
\end{aligned}
$$

and define $\hat{K}_{D}^{\lambda}(d y, x)$ similarly with respect to $\hat{X}$. Hunt $[5$, III, $\S 21]$ proved the basic relationship

$$
K_{D}^{\lambda} V^{\lambda}=V^{\lambda} \hat{K}_{D}^{\lambda}
$$

Finally, a family of random variables $\{C(t) ; t \geqq 0\}$ is a c.a.f. of $(X, T)$ if (i), (ii), and (iii) above are satisfied with $\sigma$ replaced by $T$ in (i) and $\mathscr{F}_{t}^{*}$ replaced by $\mathscr{F}_{t}^{*} \cap \mathscr{F}_{T}=\mathscr{F}_{\min (T, t)} \cap \mathscr{F}^{*}$.

Let $D_{n}=\left\{x: \hat{E}^{x}\left(e^{-T}\right)<1-1 / n\right\}$. Then $\hat{B}=\bigcup D_{n}$ and each $D_{n}$ is cospecial. Recall [5, III, p. 205] that a set is special if it is a finely open analytic set with compact closure and is contained in $\left\{x: E^{x}\left(e^{-T}\right)<\beta\right\}$ for some $\beta<1$. Cospecial sets are defined similarly with respect to $\hat{X}$ and $T$. Furthermore each $D_{n}$ is a cofinely open Borel set and if $T_{n}=T_{D_{n}^{c}}$ then $\hat{P}^{x}\left(T_{n} \rightarrow T\right)=1$ for each $x$. 
LEMMA 4.1. $\quad P^{x}\left(T_{n} \rightarrow T\right.$ as $\left.n \rightarrow \infty\right)=1$ for almost all $x$.

Proof. Let $K_{n}=K_{D_{n}^{c}}$ and $\hat{K}_{n}=\hat{K}_{D_{n}^{c}}$; then

$$
\hat{E}^{z}\left(T-T_{n} ; T_{n}<T\right)=\int\left[\int d x V(x, y)\right] \hat{K}_{n}(d y, z) .
$$

Therefore

$$
\begin{aligned}
0 & =\lim _{n \rightarrow \infty} \int d z \int\left[\int d x V(x, y)\right] \hat{K}_{n}(d y, z) \\
& =\lim _{n \rightarrow \infty} \int d x\left\{\int K_{n}(x, d y) \int V(y, z) d z\right\} .
\end{aligned}
$$

But $\int V(y, z) d z$ is bounded away from zero on special sets [5, II, Proposition 12.1], while the expression in braces equals $E^{x}\left(T-T_{n} ; T_{n}<T\right)$ and is therefore decreasing in $n$. Thus if $D$ is a special set $K_{n}(x, D) \rightarrow 0$, a.e. as $n \rightarrow \infty$. Let $B_{k}=\left\{x: E^{x}\left(e^{-T}\right)<1-1 / k\right\}$, so that each $B_{k}$ is special and $\bigcup B_{k}=B$. Let $R_{k}=T_{B_{k}^{c}}$. If $x$ is in $B_{k}$ then $X\left(T_{n}\right)$ is in $B_{k}$ a.s. $P^{x}$ on $\left\{T_{n}<R_{k}\right\}$. Therefore since $P^{x}\left(X\left(T_{n}\right) \in B_{k} ; T_{n}<T\right)=K_{n}\left(x, B_{k}\right) \rightarrow 0$ as $n \rightarrow \infty$ for each $k$ and almost all $x$, it follows that $P^{x}\left(\lim _{n \rightarrow \infty} T_{n} \geqq R_{k}\right)=1$ for almost all $x$ in $B_{k}$ for each $k$. Now using the fact that $P^{x}\left(R_{k} \rightarrow T\right)=1$ for each $x$ we find that $P^{x}\left(T_{n} \rightarrow T\right.$ as $\left.n \rightarrow \infty\right)=1$ for almost all $x$ in $B$. Of course, if $x$ is in $B^{c}$ then $P^{x}(T=0)=1$, and we always have $T_{n} \leqq T$ since $D_{n} \subset \hat{B} \subset \bar{G}$. This completes the proof of Lemma 4.1.

Lemma 4.2. Let $\{C(t) ; t \geqq 0\}$ be a c.a.f. of $(X, T)$ and suppose that $\phi(x)=E^{x}[C(T)]$ is finite for all $x$. If $K_{n}=K_{D_{n}^{c}}$ where the $D_{n}$ are defined above, then $K_{n} \phi \rightarrow 0$ a.e. and $K_{D_{n}} \phi \uparrow \phi$ everywhere.

Proof. A simple calculation yields

$$
K_{n} \phi(x)=E^{x}\left\{C(T)-C\left(T_{n}\right)\right\},
$$

and so the first statement follows from Lemma 4.1 and the continuity of $C$. Concerning the second statement, since $P^{x}(T>0)=1$ for all $x$ in $B$, it follows from Lemma 4.1 that almost all $x$ in $B$ are regular for some $D_{n}$ and for such an $x$ and $n$ we have $K_{D_{n}} \phi(x)=\phi(x)$. Since $K_{D_{n}} \phi(x)=E^{x}\left\{C(T)-C\left(T_{D_{n}}\right)\right\}$ is increasing in $n$, the last sentence implies that $K_{D_{n}} \phi \uparrow \phi$ almost everywhere on $B$. But both $\phi$ and $\lim _{n \rightarrow \infty} K_{D_{n}} \phi$ being $(X, T)$ excessive we must have $K_{D_{n}} \phi \uparrow \phi$ everywhere on $B$ and hence everywhere since $\phi$ and $K_{D_{n}} \phi$ vanish off $B$.

The next theorem is the main result of this section.

THEOREM 4.3. Let $\{C(t) ; t \geqq 0\}$ be a c.a.f. of $(X, T)$ and suppose that $\phi(x)=E^{x}\{C(T)\}$ is finite for all $x$. Then there exists a unique measure $\mu$ carried by $B$ and finite on cospecial sets such that $\phi=V \mu$. 
Proof. The measure $v(d x)=\phi(x) d x$ is excessive relative to $(\hat{X}, T)$ and so according to Theorem 14.6 of Hunt [5, II], when interpreted in the present situation, we can write $\phi=V \mu+\psi$ where $\mu$ is a measure in $\mathscr{N}$ (hence finite on cospecial sets) and $\psi$ is excessive relative to $(X, T)$. See Hunt [5, III, p. 206] for the definition of $\mathscr{N}$. Moreover, if $\eta(d x)=\psi(x) d x$ then the operator $\hat{M}_{D}$ in Theorem 14.6 of $[5, \mathrm{II}]$ is given by $\hat{M}_{D} \eta(d x)=K_{D} \psi(x) d x$. Therefore $\psi$ satisfies $K_{n} \psi=\psi$ for all $n$ where $K_{n}=K_{D_{n}^{c}}$ and $D_{n}$ are those defined above. Since $V \mu \geqq 0$ we have $\psi \leqq \phi$ and so $\psi=K_{n} \psi \leqq K_{n} \phi$. Using Lemma 4.2 this implies that $\psi=0$ a.e., and hence everywhere since $\psi$ is excessive. Thus $\phi=V \mu$. The uniqueness is a consequence of the fact that a measure in $\mathscr{N}$ is determined by its potential. Thus Theorem 4.3 is established.

The next result is essentially due to Meyer [8, Theorem 6.4, p. 218]. The proof given here, although less general, is much simpler than Meyer's.

THEOREM 4.4. Let $\{C(t) ; t \geqq 0\}$ be as in Theorem 4.3. If $h$ is a non-negative (measurable) function, then

$$
E^{x} \int_{0}^{T} h\left(X_{t}\right) d C(t)=V(h \mu)(x)=\int V(x, y) h(y) \mu(d y),
$$

where $\mu$ is the measure corresponding to $C$ constructed in Theorem 4.3.

Proof. It is enough to establish (4.2) when $h=I_{F_{1}}$, where $F_{1}$ is an open subset of $E$ whose boundary has $\mu$ measure zero and $I_{F_{1}}$ denotes the indicator (characteristic) function of $F_{1}$. Let $F_{2}=E-F_{1}$ and set $h_{i}=I_{F_{i}}, i=1,2$, and

$$
\phi_{i}(x)=E^{x} \int_{0}^{T} h_{i}\left(X_{t}\right) d C(t), \quad i=1,2 .
$$

Since $t \rightarrow \int_{0}^{T \wedge t} h_{i}\left(X_{s}\right) d C(s)$, where $T \wedge t=\min (T, t)$, is a c.a.f. of $(X, T)$ and $\phi_{i} \leqq \phi$, it follows from Theorem 4.3 that there exist measures $\mu_{i}$ such that $\phi_{i}=V \mu_{i}$. Because $\phi=\phi_{1}+\phi_{2}$ the uniqueness of $\mu$ implies that $\mu=\mu_{1}+\mu_{2}$. Thus $\mu_{i}$ is absolutely continuous with respect to $\mu$ and if we let $d \mu_{i}=f_{i} d \mu$, then $f_{1}+f_{2}=1[\mu]$. Since $C$ is continuous

$$
K_{F_{i}} \phi_{i}(x)=E^{x} \int_{T_{F_{i}}}^{T} h_{i}\left(X_{t}\right) d C(t)=\phi_{i}(x),
$$

and hence $\phi_{i}=K_{F_{i}} V \mu_{i}=V \hat{K}_{F_{i}} \mu_{i}$. Therefore $\mu_{i}=\hat{K}_{F} \mu_{i}$, and this implies that $\mu_{i}$ is concentrated on $F_{i}$. Using the fact that the $\mu$ measure of the boundary of $F_{i}$ is zero we see that $f_{i}=h_{i}[\mu]$ and so $d \mu_{i}=h_{i} d \mu$. But this is what we wanted to prove.

CoRollary 4.5. If $D$ is semi-polar then $\mu(D)=0$. Here $\mu$ is the measure constructed in Theorem 4.3.

Proof. It suffices to show that $\mu(D)=0$ when $D$ is a thin Borel set. But in 
this case $X(t)$ is in $D$ for only countably many values of $t$. See Hunt [5, I, Proposition 2.4]. Therefore using Theorem 4.4 and the continuity of $C$ we obtain

$$
0=E^{x} \int_{0}^{T} I_{D}\left(X_{t}\right) d C(t)=\int_{D} V(x, y) \mu(d y),
$$

and this yields $\mu(D)=0$.

A consequence of this corollary is that $\mu$ is concentrated on $B \cap \hat{B}$ since $\hat{B}-B$ is semi-polar. Thus we have shown that if $\phi$ is the finite potential of a c.a.f. $\{C(t) ; t \geqq 0\}$ of $(X, T)$ then $\phi=V \mu$ where $\mu$ is a measure concentrated on $B \cap \hat{B}$, charging no semi-polar set, and finite on cospecial sets. Moreover $\mu$ is unique and (4.2) holds whenever $h \geqq 0$. Recall that the potential of $C$ is just the function $\phi(x)=E^{x}[C(T)]$.

5. Smooth additive functionals. The previous assumptions on $X$ and $\hat{X}$ remain in force. A smooth additive functional (s.a.f.) of $X$ is a continuous additive functional, $A$, which satisfies the following finiteness condition: Whenever $G$ is an exit set, $A\left(T_{G^{\prime}}\right)$ is a.s. finite. It will be seen later on that if $A$ is an s.a.f. then $A(t)$ is a.s. finite on $\{t<\sigma\}$. This section and the following one are devoted to setting up a one-to-one correspondence between smooth additive functionals and a certain class of measures on $E$.

Let $A=\{A(t) ; t \geqq 0\}$ be an s.a.f. of $X$ and let $G$ be a fixed exit set. Let $T=T_{G}$, and set

$$
\begin{aligned}
& g(x)=E^{x}\left\{e^{-A(T)}\right\}, \\
& C(t)=\int_{0}^{t \wedge T} g[X(s)] d A(s), \quad t \wedge T=\min (t, T) .
\end{aligned}
$$

Since $A(T)$ is a.s. finite we see that $0<g(x) \leqq 1$, and it is not difficult to see that $1-g$ is excessive relative to $(X, T)$. Clearly $t \rightarrow C(t)$ is a c.a.f. of $(X, T)$ and a routine calculation using the right continuity of $t \rightarrow g\left(X_{t}\right)$ on $[0, T)$ yields

$$
\begin{aligned}
\phi(x) & =E^{x}\{C(T)\}=E^{x} \int_{0}^{T} \exp \{-[A(T)-A(s)]\} d A(s) \\
& =E^{x}\left[1-e^{-A(T)}\right]=1-g(x),
\end{aligned}
$$

and so $\phi \leqq 1$. The results of $\S 4$ imply the existence of a unique measure $v$ concentrated on $B \cap \hat{B}$, charging no semi-polar set, and finite on cospecial sets such that $\phi=V v$. Here $B$ and $\hat{B}$ have the same meetings as in $\S 4$. Define

$$
\mu(D)=\int_{D}[g(x)]^{-1} v(d x)
$$

Since $0<g \leqq 1$ the measures $\mu$ and $v$ are equivalent and, according to Theorem 4.4 , if $f \geqq 0$ we have 


$$
\begin{aligned}
V(f \mu)(x) & =V[(f / g) v](x) \\
& =E^{x} \int_{0}^{T} f\left(X_{t}\right) d A(t) .
\end{aligned}
$$

Finally there exists at least one strictly positive $f$ (namely $g$ ) for which the above integrals are bounded in $x$.

THEOREM 5.1. There exists an increasing sequence $\left\{B_{n}\right\}$ of finely open Borel sets such that

(i) $B=\bigcup B_{n}$.

(ii) $\int_{B_{n}} V(x, y) \mu(d y)$ is bounded in $x$ for each $n$. Here $V$ is the potential kernel for $(X, T)$.

(iii) $P^{x}\left(T_{B_{n}^{c}}=T\right) \rightarrow 1$ as $n \rightarrow \infty$ for all $x$.

Proof. Define $B_{n}=B \cap\{g>1 / n\}$ where $g$ is defined in (5.1). Since $g$ is Borel measurable and strictly positive it is clear that $\left\{B_{n}\right\}$ is an increasing sequence of finely open Borel sets whose union is $B$. Also

$$
\begin{aligned}
\int_{B_{n}} V(x, y) \mu(d y) & \leqq n \int_{B_{n}} V(x, y) g(y) \mu(d y) \\
& \leqq n V v(x) \leqq n
\end{aligned}
$$

so that (ii) holds. As to (iii), let $T_{n}=T_{B_{n}^{c}} R=\lim T_{n}$, and for a fixed $x$ let $\alpha=P^{x}\left(T_{n}<T\right.$ for all $\left.n\right)$. Recalling that $\phi(x)=E^{x}[C(T)]=1-g(x)$, we see that $\phi\left[X\left(T_{n}\right)\right] \geqq 1-1 / n$ a.s. on $\left\{T_{n}<T\right\}$ since $X\left(T_{n}\right) \in B_{n}^{c}$ a.s. Therefore

$$
\begin{aligned}
\left(1-\frac{1}{n}\right) \alpha & \leqq E^{x}\left\{\phi\left[X\left(T_{n}\right) ; T_{n}<T\right\}=E^{x}\left[C(T)-C\left(T_{n}\right)\right]\right. \\
& \rightarrow E^{x}[C(T)-C(R)]=E^{x}\{\phi[X(R)] ; R<T\} .
\end{aligned}
$$

Because $P^{x}(R<T) \leqq \alpha$ and $\phi$ is strictly less than one it now follows that $\alpha=0$. Thus (iii) is established.

So far we have associated with each exit set $G$ a measure $\mu$ concentrated on $B \cap \hat{B}$ and for which (5.3) holds. Moreover $\mu$ is the unique measure concentrated on $B \cap \hat{B}$ such that (5.3) holds-this follows since $v(d x)=g(x) \mu(d x)$ is unique and $g>0$. Since $E$ can be covered by exit sets (Theorem 3.2) we may define a unique measure $m$ on $E$ with the property that for each exit set $G$ with associated measure $\mu$ we have $\mu(D)=m(D \cap B)=m(D \cap \hat{B})$ for all Borel sets $D \subset E$, once the following compatibility relationship is established.

THEOREM 5.2. If $G_{1}$ and $G_{2}$ are exit sets with associated measures $\mu_{1}$ and $\mu_{2}$, then $\mu_{1}(D)=\mu_{2}(D)$ whenever $D \subset B_{1} \cap B_{2}$.

Proof. Let $T_{i}=T_{G_{i}^{\prime}}, V_{i}$ be the potential kernel of $\left(X, T_{i}\right)$, and $K_{D}^{i}$ be the hitting distribution of $D$ by $\left(X, T_{i}\right), i=1,2$. Let $B=B_{1} \cap B_{2}$ and $T=T_{B c}$ so 
that $T=T_{1} \wedge T_{2}=\min \left(T_{1}, T_{2}\right)$, a.s. If $W$ denotes the potential kernel for $(X, T)$, then it is not difficult to see that for each fixed $x$ in $B$

$$
W(x, y)=V_{i}(x, y)-\int K_{B^{c}}^{i}(x, d z) V_{i}(z, y)
$$

with the possible exception of a semi-polar set of $y$ (one can actually show that the exceptional set of $y$ is polar), $i=1,2$. Let $h$ be a function that is strictly positive on $B_{1} \cup B_{2}$ and such that

$$
E^{x} \int_{0}^{T_{i}} h\left(X_{t}\right) d A(t)
$$

is bounded in $x$ for $i=1,2$. Such an $h$ is easily constructed from $g_{1}$ and $g_{2}$. Since $\mu_{i}$ does not charge semi-polar sets, a simple calculation using Theorem 4.4 yields (recall that $T=T_{1} \wedge T_{2}$ a.s.).

$$
\begin{aligned}
\int_{B} W(x, y) h(y) \mu_{i}(d y)= & E^{x} \int_{0}^{T_{i}}\left(h I_{B}\right)\left(X_{t}\right) d A(t) \\
& -\int K_{B^{c}}^{i}(x, d z) E^{z} \int_{0}^{T_{t}}\left(h I_{B}\right)\left(X_{t}\right) d A(t) \\
= & E^{x} \int_{0}^{T_{1} \wedge T_{2}}\left(h I_{B}\right)\left(X_{t}\right) d A(t) .
\end{aligned}
$$

Therefore $h d \mu_{1}=h d \mu_{2}$ on $B$ since they have the same potential, and hence $\mu_{1}=\mu_{2}$ on $B$. This completes the proof of Theorem 5.2.

Thus, as explained above Theorem 5.2, we can associate with each s.a.f. $A$ of $X$ a measure $m$ on $E$ with the following properties:

(i) $m$ charges no semi-polar set.

(ii) If $G$ is an exit set and $f \geqq 0$ then $E^{x} \int_{0}^{T} f\left(X_{t}\right) d A(t)=\int_{B} V(x, y) f(y) m(d y)$ where $T, B$, and- $V$-have their usual meanings with regard to $G$.

(iii) If $G$ is an exit set, then there exists an increasing sequence $\left\{B_{n}\right\}$ of finely open Borel sets whose union is $B$, such that $\int_{B_{n}} V(x, y) m(d y)$ is bounded in $x$ for each $n$ and $P^{x}\left(T_{B_{n}^{c}}=T\right) \rightarrow 1$ as $n \rightarrow \infty$ for each $x$.

DEFINITION. A measure $\mu$ on $E$ satisfying (i) and (iii) above is said to be smooth (relative to $X$ ).

Let us now prove the assertion made at the beginning of this section.

THEOREM 5.3. If $A$ is an s.a.f. then $t \rightarrow A(t)$ is a.s. finite on the interval $[0, \sigma)$.

Proof. Let $R=\inf \{t: A(t)=\infty\}$ and assume that there exists an $x$ in $E$ such that $P^{x}(R<\sigma)>0$; then there exists an exit set $G$ such that $P^{x}(X(R) \in G ; R<\sigma)>0$. Let $R_{n}=\inf \{t: A(t)=n\}$. Then $R_{n} \uparrow R$ and $R_{n}<R$ on $\{R<\sigma\}$. Now $\lim _{t \uparrow R} X(t)$ exists $P^{x}$ a.s. on $\{R<\sigma\}$ and since $X\left(R_{n}\right) \rightarrow X(R), R_{n}<R, P^{x}$ a.s. on $\{R<\sigma\}$, it follows that $\lim _{t \uparrow R} X(t)=X(R)$ a.s. $P^{x}$ on $\{R<\sigma\}$. But $G$ is open 
and so there exists an integer $n$ such that $P^{x}\left\{X(t) \in G\right.$ for all $\left.t \in\left[R_{n}, R\right]\right\}>0$. Using $A(R)=A\left(R_{n}\right)+A\left(R-R_{n}, \theta_{R_{n}}\right), \quad A\left(R_{n}\right)=n$, and $R-R_{n}=R\left(\theta_{R}\right)$ we find that

$$
\begin{aligned}
0 & =P^{x}\left\{A\left(R-R_{n}, \theta_{R_{n}}\right)<\infty ; X(t) \in G \text { for all } t \in\left[R_{n}, R\right]\right\} \\
& =E^{x}\left\{P^{X\left(R_{n}\right)}(A(R)<\infty ; X(t) \in G \text { for all } t \in[0, R])\right\} \\
& >0
\end{aligned}
$$

since $R<T_{G^{c}}$ and hence $A(R)<\infty$ on $\{X(t) \in G$ for all $t \in[0, R]\}$. Thus Theorem 5.3 is established.

THEOREM 5.4. The s.a.f. $A$ is strictly increasing on the interval $[0, \sigma)$ if and only if the corresponding measure $m$ is strictly positive on nonempty finely open Borel sets.

Proof. Suppose $A$ is strictly increasing and that $D$ is a nonvoid finely open Borel set. We may assume $D \subset G$ where $G$ is an exit set (replace $D$ by $D \cap G$ ). With our usual notation ( $T=T_{G^{\prime}}$, etc.) we have for $x \in D \subset G$ that

$$
0<E^{x} \int_{0}^{T} I_{D}[X(t)] d A(t)=\int_{D} V(x, y) m(d y),
$$

and so $m(D) \neq 0$. Conversely let $R=\inf \{t: A(t)>0\}$; then since $A$ is finite on $[0, \sigma)$ the assertion that $A$ is strictly increasing on $[0, \sigma)$ is equivalent to $P^{x}(R>0)=0$ for all $x$. Hence, using the zero-one law, we must show that $D=\left\{x: P^{x}(R>0)=1\right\}$ is empty.

We will first show that $D$ is a Borel set. Let $\left\{G_{i}\right\}$ be a sequence of exit sets forming a basis for the topology of $E$. Let

$$
f(x)=\sup _{i} \lim _{\lambda \rightarrow \infty} I_{G_{i}}(x) E^{x}\left\{\exp \left[-\lambda A\left(T_{G_{i}^{\prime}}\right)\right]\right\}
$$

and let $T=\inf \{t: X(t) \neq X(0)\}$. According to the comments following (5.1) the function $f$ is Borel measurable. Clearly if $x$ is not in $D$ then $f(x)=0$. If $x$ is in $D$ and $P^{x}(T=0)=1$ then it is equally clear that $f(x)=1$. If $x$ is in $D$ and $P^{x}(T=0)=0$, then it is not difficult to see that $P^{x}[A(T)=0]=1$. But for such an $x, P^{x}[X(T)=X(0)]=0$, and so it follows that $f(x)=1$. Thus $D=\{f=1\}$ and hence is a Borel set.

If $x_{0}$ is in $D$ let $G$ be an exit set containing $x_{0}$ and set $F=D \cap G$ so that $F$ is nonempty. Let $S$ be the first hit of $F^{c}$. We first assert that $P^{x}(R<S)=0$ for all $x$ in $B$. We are using our usual notation with regard to the exit set $G$. If for some $x$ in $B, P^{x}(R<S)>0$, then using the fact that $A(R)=0$, we have for all $t>0$

$$
\begin{aligned}
0 & <P^{x}(R<S)=P^{x}[A(R+t)>0 ; R<S] \\
& =E^{x}\left\{P^{X(R)}(A(t)>0) ; R<S\right\} .
\end{aligned}
$$


But $X(R) \in F$, a.s. on $\{R<S\}$ and $A(t)>0$ implies $R<t$, so that this last expression approaches zero as $t \rightarrow 0$ which is a contradiction. Also one easily sees that $F$ is finely open. Let $H=F^{c}$ and as usual let $T=T_{G^{\prime}}, g(x)=E^{x}\left\{e^{-A(T)}\right\}$, $d \mu=g d m$; then since $P^{x}(R<S)=0$ we have

$$
\begin{aligned}
V \mu(x) & =E^{x} \int_{0}^{T} g[X(t)] d A(t) \\
& =E^{x}\left\{\int_{S}^{T} g[X(t)] d A(t) ; S<T\right\} \\
& =K_{H} V \mu(x)=V \hat{K}_{H} \mu(x) .
\end{aligned}
$$

Therefore $\mu=\hat{K}_{H} \mu$ or, recalling that $H=F^{c}, \mu$ assigns no mass to the set of points of $F$ that are not coregular for $F^{c}$. Since $F$ is finely open, Theorem 2.1 implies that the set of points in $F$ that are coregular for $F^{c}$ is semi-polar. Hence $\mu(F)=0$ and so $m(F)=0$. Since $F$ is finely open this implies $F$ is empty which is a contradiction. Thus Theorem 5.4 is established.

6. Smooth measures. In $\S 5$ we associated with each s.a.f. of $X$ a unique smooth measure. In this section we will begin with a smooth measure and show that it corresponds to an s.a.f. of $X$. Thus in the remainder of this section $m$ is a given smooth measure on $E$.

To begin the construction of the desired additive functional let $G$ be an exit set and let $B, \hat{B}, T, V$, etc. have their usual meanings. The following theorem may be deduced by combining Theorems 2.5 and 6.5 of Meyer [8, part II]. However, we will give a direct proof that in our opinion is both simpler and more natural.

THEOREM 6.1. Let $\mu$ be a measure that charges no semi-polar set and such that $\phi=V \mu$ is bounded. If $\left\{D_{n}\right\}$ is an increasing sequence of Borel subsets of $B, T_{n}=T_{D_{n}^{c}}, R=\lim T_{n}$, then for all $x$

$$
\lim _{n} E^{x}\left\{\phi\left[X\left(T_{n}\right)\right] ; T_{n}<T\right\}=E^{x}\{\phi[X(R)] ; R<T\} .
$$

Proof. Since $R$ is a stopping time the zero-one law implies that $P^{x}(R>0)$ is either zero or one. Let $F=\left\{x: P^{x}(R>0)=1\right\}$. Since the conclusion is obvious if $x$ is not in $F$ we may assume $x$ in $F$.

Let $f$ and $h$ denote bounded non-negative functions, and let $K_{n}=K_{D_{n}^{c}}$ and similarly for $\hat{K}_{n}$. If we define

$$
\phi_{n}(x)=\int f(z) d z \int K_{n}(z, d y) V(y, x),
$$

then each $\phi_{n}$ is excessive relative to $(\hat{X}, T)$. Also 


$$
\begin{aligned}
\phi_{n}(x) & =\int f(z) d z \int V(z, y) \hat{K}_{n}(d y, x) \\
& =E^{x}\left\{\int_{T_{n}}^{T} f\left(X_{t}\right) d t ; T_{n}<T\right\},
\end{aligned}
$$

so that $\phi_{n}$ decreases as $n$ increases. If $q=\lim \phi_{n}$, then $q$ is cosuper mean valued being the limit of a decreasing sequence of coexcessive functions. Thus by Doob's Theorem (Theorem 2.2) $q$ differs from its coexcessive regularization $q^{*}$ on at most a semi-polar set. Moreover

$$
\begin{aligned}
\lim _{n} \int \phi_{n}(x) h(x) d x & =\lim _{n} \int f(y) E^{y}\left\{\int_{T_{n}}^{T} h\left(X_{t}\right) d t ; T_{n}<T\right\} d y \\
& =\int f(y) E^{y}\left\{\int_{R}^{T} h\left(X_{t}\right) d t ; R<T\right\} d y \\
& =\int f(y) d y \int K_{R}(y, d z) \int V(z, x) h(x) d x,
\end{aligned}
$$

where $K_{R}(x, A)=P^{x}[X(R) \in A ; R<T]$. On the other hand $\lim _{n} \int \phi_{n}(x) h(x) d x$ $=\int q(x) h(x) d x$, and so

$$
q(x)=\int f(y) d y \int K_{R}(y, d z) V(z, x)
$$

for almost all $x$. Therefore $q^{*}$ must equal the right-hand side of (6.1) a.e. and hence everywhere since $q^{*}$ and the right-hand side of (6.1) are both coexcessive. This in turn implies that (6.1) must hold except on a semi-polar set. But $\mu$ charges no semi-polar set and so we obtain

$$
\begin{aligned}
\lim _{n} \int\left(\int f(z) d z \int K_{n}(z, d y) V(y, x)\right) \mu(d x) \\
\quad=\int\left(\int f(z) d z \int K_{R}(z, d y) V(y, x)\right) \mu(d x) .
\end{aligned}
$$

Since $V \mu$ is excessive it follows that $K_{n} V \mu$ decreases with $n$ and that $K_{n} V \mu \geqq K_{R} V \mu$ for all $n$. However, if $\psi=\lim _{n} K_{n} V \mu$ what we have shown above implies that $\psi=K_{R} V \mu$ a.e. Let $A$ be the exceptional set on which $\psi \neq K_{R} V \mu$; then if $x$ is in $B$ let $\left\{t_{j}\right\}$ be a sequence decreasing to zero such that $P^{x}\left[X\left(t_{j}\right) \in A\right]=0$ for all $j$. This is possible since $\xi(A)=0$ implies that $P_{t}(x, A)=0$ for almost all $t$. Now (recall $\phi=V \mu$ )

$$
\begin{aligned}
& E^{x}\left\{K_{n} V \mu\left[X\left(t_{j}\right)\right] ; t_{j}<T\right\} \\
& \quad \geqq E^{x}\left\{E^{X\left(t_{j}\right)}\left\{\phi\left[X\left(T_{n}\right)\right] ; T_{n}<T\right\} ; t_{j}<T_{n}\right\} \\
& \quad=E^{x}\left\{\phi\left[X\left(T_{n}\right)\right] ; T_{n}<T ; t_{j}<T_{n}\right\},
\end{aligned}
$$


where the Markov property was used in obtaining the last equality. But this last expression is equal to

$$
E^{x}\left\{\phi\left[X\left(T_{n}\right)\right] ; T_{n}<T\right\}-E^{x}\left\{\phi\left[X\left(T_{n}\right)\right] ; T_{n}<T ; T_{n} \leqq t_{j}\right\}
$$

and combining these facts we find

$$
\begin{aligned}
E^{x}\left\{K_{n} \phi\left[X\left(t_{j}\right)\right] ; t_{j}\right. & <T\}+E^{x}\left\{\phi\left[X\left(T_{n}\right)\right] ; T_{n} \leqq t_{j}\right\} \\
& \geqq E^{x}\left\{\phi\left[X\left(T_{n}\right)\right] ; T_{n}<T\right\} \geqq \psi(x) .
\end{aligned}
$$

On the other hand if we let $n \rightarrow \infty$ the first term on the left-hand side of (6.2) approaches

$$
E^{x}\left\{K_{R} \phi\left[X\left(t_{j}\right)\right] ; t_{j}<T\right\}
$$

by the choice of $t_{j}$, while the second term is dominated by

$$
(\sup \phi) P^{x}\left[T_{n} \leqq t_{j}\right] \rightarrow(\sup \phi) P^{x}\left(R \leqq t_{j}\right) .
$$

If we now let $t_{j} \rightarrow 0$, then $P^{x}\left(R \leqq t_{j}\right) \rightarrow 0$ if $x$ is in $F=\left\{x: P^{x}(R>0)=1\right\}$. Also it is easily seen that $K_{R} \phi$ is super mean valued with respect to $(X, T)$ and so $\lim _{j} E^{x}\left\{K_{R} \phi\left(X_{t}\right) ; t_{j}<T\right\} \leqq K_{R} \phi(x)$. Combining these statements with (6.2) we finally find that $K_{R} \phi(x) \geqq \psi(x)$ for all $x$ in $F$. Hence $K_{R} \phi=\psi$ on $F$ since we already knew that $\psi \geqq K_{R} \phi$. Recalling that $\psi(x)=\lim _{n} K_{n} \phi(x)$ we obtain the desired conclusion of Theorem 6.1.

Let $G$ continue to denote a fixed exit set. Since $m$ is smooth there exists an increasing sequence $\left\{B_{n}\right\}$ of finely open Borel sets whose union is $B$ and such that $\phi_{n}(x)=\int_{B_{n}} V(x, y) m(d y)$ is bounded and $P^{x}\left(T_{B_{n}^{c}}=T\right) \rightarrow 1$ for all $x$.

THEOREM 6.2. There is a continuous additive functional $A$ of $(X, T)$ with $A(T)$ finite a.s., and such that for each $f \geqq 0$ we have

$$
E^{x} \int_{0}^{T} f\left(X_{t}\right) d A(t)=\int V(x, y) f(y) m(d y) .
$$

Proof. Let us define $\mu_{n}(D)=m\left(D \cap B_{n}\right)$ so that each $\mu_{n}$ is a measure which charges no semi-polar set. Clearly $V \mu_{n}=\phi_{n}$ which is bounded. Therefore Theorem 6.1 applies to $\phi_{n}$. It is by now a standard fact due to Šr [9] (see also Proposition 5.2 and Theorem 5.3 of [3]) that because of this there exists a c.a.f. $A^{n}$ of $(X, T)$ such that $E^{x}\left\{A^{n}(T)\right\}=\phi_{n}(x)=V \mu_{n}(x)$. Theorem 4.4 then implies that

$$
E^{x} \int_{0}^{T} f\left(X_{t}\right) d A^{n}(t)=\int V(x, y) f(y) \mu_{n}(d y)
$$

for all $f \geqq 0$.

Let $T_{n}=T_{B_{n}^{c}}, K_{n}=K_{B_{n}^{c}}, \hat{K}_{n}=\hat{K}_{B_{n}^{c}}$; then if $k \geqq n$

$$
E^{x}\left\{A^{k}\left(T_{n}\right)\right\}=V \mu_{k}(x)-V \hat{K}_{n} \mu_{k}(x) \text {. }
$$

But 


$$
\begin{aligned}
\hat{K}_{n} \mu_{k}(D) & =\int_{B_{n}} \hat{K}_{n}(D, y) \mu_{k}(d y)+\int_{B_{n}^{\mathrm{c}}} \hat{K}_{n}(D, y) \mu_{k}(d y) \\
& =\hat{R}_{n} \mu_{n}(D)+\mu_{k}\left(D \cap B_{n}^{c}\right),
\end{aligned}
$$

since the set of points in $B_{n}^{c}$ not coregular for $B_{n}^{c}$ is semi-polar. It is now immediate from (6.4) that $E^{x}\left\{A^{k}\left(T_{n}\right)\right\}=E^{x}\left\{A^{n}\left(T_{n}\right)\right\}$ for all $x$ and so the uniqueness theorem for c.a.f. (see Meyer [8, Theorem 4.5, part II]) implies that $A^{n}(t)$ and $A^{k}(t)$ agree a.s. on the interval $\left[0, T_{n}\right]$. However $P^{x}\left(T_{n}=T\right) \rightarrow 1$ as $n \rightarrow \infty$. Therefore we can define $A(t)=\lim _{n \rightarrow \infty} A^{n}(t)$ and obtain a finite (since $A^{n}(T)$ is finite and $T_{n}=T$ a.s. for sufficiently large $n)$ continuous additive functional of $(X, T)$.

If we let $n \rightarrow \infty$ in (6.3) and use Fatou's lemma we obtain

$$
E^{x}\left\{\int_{0}^{T} f\left(X_{t}\right) d A(t)\right\} \leqq \int V(x, y) f(y) m(d y)
$$

for all $f \geqq 0$. To obtain the reverse inequality let $0 \leqq f \leqq 1$ and let $f$ vanish off $B_{n}$; then $V(f m)=V\left(f \mu_{k}\right)$ for all $k \geqq n$. Using (6.3) we find that

$$
\begin{aligned}
V\left(f \mu_{k}\right)(x) & =E^{x} \int_{0}^{T_{k}} f[X(t)] d A^{k}(t)+E^{x} \int_{T_{k}}^{T} f\left(X_{t}\right) d A^{k}(t) \\
& \leqq E^{x} \int_{0}^{T} f[X(t)] d A(t)+K_{k} V\left(f \mu_{k}\right) .
\end{aligned}
$$

But $K_{k} V\left(f \mu_{k}\right) \leqq K_{k} V \mu_{n} \rightarrow 0$ as $k \rightarrow \infty$ since $V \mu_{n}$ is the bounded potential of the c.a.f. $A^{n}$. Thus we obtain the conclusion of the theorem for any $f$ vanishing off some $B_{n}$ and hence for any $f$ vanishing off $B$, but this suffices to establish Theorem 6.2.

We are now in a position to construct an s.a.f. $A$ of $X$ such that the smooth measure corresponding to $A$ is $m$.

THEOREM 6.3. There exists a s.a.f. $A$ of $X$ such that for each exit set $G$ and $f \geqq 0$ we have

$$
E^{x} \int_{0}^{T} f[X(t)] d A(t)=\int V(x, y) f(y) m(d y),
$$

where $T$ and $V$ have their usual meanings with regard to $G$.

Proof. Let $\left\{G_{j}\right\}$ be a covering of $E$ by exit sets and let $N$ be a Borel measurable function from $E$ to the positive integers such that: (i) $x \in G_{N(x)}$ for all $x$; (ii) $\rho\left(x, G_{N(x)}^{c}\right)$ is bounded away from zero on compacts where $\rho$ is the metric for $E$. Theorem 3.2 shows the existence of $\left\{G_{j}\right\}$ and $N$. As usual let $T_{j}=T_{G^{\prime}}$, and let $V_{j}$ denote the potential kernel for $\left(X, T_{j}\right)$. Also let $A_{j}$ be the c.a.f. of $\left(X, T_{j}\right)$ constructed in Theorem 6.2. Recall that $A_{j}\left(T_{j}\right)$ is a.s. finite. We intend to piece the $A_{j}$ together in order to obtain $A$. 
Define $T=T_{N[X(0)]}$ if $X(0) \in E$ and 0 otherwise, and then let

$$
\begin{aligned}
R_{0} & =0, \\
R_{n+1} & =R_{n}+T\left(\theta_{R_{n}}\right), \quad n \geqq 0 .
\end{aligned}
$$

It is easy to see that $P^{x}\left[R_{n+1}=R_{n} ; R_{n}<\sigma\right]=0$ for all $x$, and from property (ii) above of the covering $\left\{G_{j}\right\}$ together with the fact that the paths have left-hand limits it follows that $P^{x}\left[R_{n} \leqq t\right.$ for all $\left.n, t<\sigma\right]=0$ for each $t$ and $x$. Consequently $R_{n} \rightarrow \sigma$ a.s. We now define

$$
A(t, \omega)=A_{\mathrm{N}[X(0, \omega)]}(t, \omega), \quad 0 \leqq t \leqq R_{1}(\omega),
$$

and having defined $A(t)$ for $t \leqq R_{n}$ we set

$$
A(t, \omega)=A\left[R_{n}(\omega), \omega\right]+A_{N\left[X\left(0, \theta_{R_{n}} \omega\right)\right]}\left[t-R_{n}(\omega), \theta_{R_{n}} \omega\right]
$$

for $R_{n}(\omega) \leqq t<R_{n+1}(\omega)$. This then defines $A(t, \omega)$ on the interval $[0, \sigma)$ as a continuous, nondecreasing function of $t$. Of course, we set $A(t, \omega)=\lim _{s} \uparrow \sigma(\omega) A(s, \omega)$ if $t \geqq \sigma(\omega)$. The assertions of the theorem are easily checked once we establish the following compatibility condition: Let $G_{1}$ and $G_{2}$ be any two exit sets (not necessarily the first two elements in our covering $\left\{G_{j}\right\}$ ) and let $T_{1}, T_{2}, V_{1}, V_{2}$, etc. have their usual meanings; then $A_{1}$ and $A_{2}$ agree a.s. on the interval $\left[0, T_{1} \wedge T_{2}\right]$. Here $A_{j}$ is the c.a.f. of $\left(X, T_{j}\right)$ constructed in Theorem $6.2 ; j=1,2$. Just as in the proof of Theorem 5.2 one can find a strictly positive function, $h$, on $B=B_{1} \cap B_{2}$ such that

$$
\int W(x, y) h(y) m(d y)=E^{x} \int_{0}^{T_{1} \wedge T_{2}} h\left(X_{t}\right) d A_{j}(t)
$$

for $j=1,2$, where $W$ is the potential kernel for the process terminated when it first leaves $B$ and $h$ is such that both expressions in (6.5) are bounded in $x$. Meyer's uniqueness theorem then implies that the c.a.f.'s $t \rightarrow \int_{0}^{t \wedge T} h\left(X_{s}\right) d A_{1}(s)$ and $t \rightarrow \int_{0}{ }^{\wedge} h\left(X_{s}\right) d A_{2}(s)$ agree a.s. on $\left[0, T_{1} \wedge T_{2}\right]$, and hence it follows that $A_{1}$ and $A_{2}$ agree a.s. on this interval. Thus Theorem 6.3 is proved.

We have now completed the task begun in $\$ 5$ of setting up a one-to-one correspondence between s.a.f. of $X$ and smooth measures. Moreover, the correspondence is such that a functional is a.s. strictly increasing on $[0, \sigma)$ if and only if the corresponding measure is strictly positive on nonvoid finely open Borel sets.

7. Processes with identical hitting distributions. As before $X=\left(E, P^{x}\right)$ denotes a Hunt process satisfying (F) with dual process $\hat{X}=\left(E, P^{x}\right)$, and, as usual, we suppose that there are no traps in $E$ for either $X$ or $X$. Let $Y=\left(E,{ }^{*} P^{*}\right)$ be another Hunt process with the same distribution of first hits as $X$, i.e., $P_{D}(x, \cdot)={ }^{*} P_{D}(x, \cdot)$ for all $x$ and whenever $D=K$ or $D=E-K$ where $K$ is 
a compact subset of $E$. Our notation is such that the symbol ${ }^{*} P$ has the same relationship to $Y$ as $P$ has to $X$. For example

$$
{ }^{*} P_{D}(x, d y)={ }^{*} P^{x}\left[X\left(T_{D}\right) \in d y ; T_{D}<\infty\right] .
$$

It was shown in [3] that there exists a unique s.a.f. $A$ of $X$ that is a.s. strictly increasing on $[0, \sigma)$ and such that if $\tau(t)$ is the function inverse to $A(t)$, i.e.,

$$
\begin{aligned}
& \tau(t)=s \text { if } t<A(\sigma) \text { and } A(s)=t, \\
& \tau(t)=\sigma \quad \text { if } t \geqq A(\sigma),
\end{aligned}
$$

then $X[\tau(t)]$ is equivalent to $Y(t)$, that is,

$$
P^{x}[X[\tau(t)] \in D]={ }^{*} P^{x}[X(t) \in D]
$$

for all Borel sets $D$ and all $x$. It follows from the results of $\S \S 5$ and 6 that there is a smooth measure $m$ corresponding to the additive functional $A$ which is strictly positive on nonvoid finely open Borel sets.

Conversely if $m$ is a smooth measure which is strictly positive on nonvoid finely open Borel sets and if $A$ is the corresponding functional then it is easy to see that $Y(t)=X[\tau(t)]$, where $\tau(t)$ is again the function inverse to $A(t)$, is a Hunt process on $E$ with the same hitting distributions as $X$. However, in general $Y$ does not satisfy hypothesis (F). Thus there is a one-to-one correspondence between smooth measures strictly positive on finely open Borel sets and Hunt processes with the same hitting distributions as $X$. If $m$ corresponds to $Y$, we will call $m$ the speed measure of $Y$. Note that the speed measure of $X$ is just the basic measure $\xi$.

Let $Y$ be a Hunt process having the same hitting distributions as $X$ and with corresponding speed measure $m$. It is easy to see that $X$ and $Y$ induce the same fine topology on $E$ and it was shown in [3] that $X$ and $Y$ have the same excessive functions. Now let $G$ be an exit set (for $X$ ) and let $T$, $V$, etc., have their usual meanings. Also let $W(x, d y)$ be the potential kernel for $(Y, T)$; then if $f \geqq 0$ we have $\left({ }^{*} E^{x}\right.$ is the expectation operator going with $\left.{ }^{*} P^{x}\right)$

$$
\begin{aligned}
W f(x) & ={ }^{*} E^{x} \int_{0}^{T} f[X(t)] d t \\
& =E^{x} \int_{0}^{A(T)} f[X(\tau(t))] d t \\
& =E^{x} \int_{0}^{T} f[X(t)] d A(t) \\
& =\int V(x, y) f(y) m(d y) .
\end{aligned}
$$


Hence $W(x, d y)=V(x, y) m(d y)$, that is, $V(x, y)$ is a density for $W(x, d y)$ with respect to the speed measure $m$.

In a certain sense the speed measure $m$ of $Y$ depends only on the basic measure $\xi$ and not on $X$. Namely let $X_{1}$ and $X_{2}$ be Hunt processes satisfying hypothesis (F) with basic measures $\xi_{1}$ and $\xi_{2}$ and with the same hitting distributions. Now if $Y$ is a Hunt process with the same hitting distributions as $X_{1}$ and hence $X_{2}$, and if $m_{j}$ is the speed measure of $Y$ relative to $X_{j}, j=1,2$, then it is easily seen that $m_{1}=m_{2}$ provided that $\xi_{2}$ is the speed measure of $X_{2}$ relative to $X_{1}$. This is the reason we call $m$ the speed measure of $Y$ rather than the speed measure of $Y$ relative to $X$. Of course, if the basic measure is changed then so are all speed measures.

If $h$ is the indicator function of $\hat{B}$, then $h$ is excessive relative to $(\hat{X}, T)$ and so there exists a sequence $\left\{f_{n}\right\}$ of positive functions on $\hat{B}$ such that $\int d y f_{n}(y) V(y, x)$ increases to $h$. Therefore if $\mu$ is the restriction of $m$ to $B \cap B$ and $d v_{n}=f_{n} d \xi$, we have for $D \subset B$

$$
v_{n} W(D)=\int_{D}\left[\int v_{n}(d y) V(y, x)\right] \mu(d x) \uparrow \mu(D),
$$

so that $\mu$ is excessive relative to $(Y, T)$ provided it satisfies the appropriate finiteness conditions.

Finally let us remark that it is an immediate consequence of the definition of a smooth measure $m$ that each point $x$ in $E$ is contained in a finely open Borel set $B$ such that if $V$ is the potential kernel for $X$ terminated when it first leaves $B$ then $V m$ is bounded.

8. Duality and smooth additive functionals. Suppose that $m$ is a smooth measure. Then it is natural to ask if $m$ is cosmooth, i.e., smooth relative to the dual process $\hat{X}$. Obviously if $X=\hat{X}$ then this is the case. Unfortunately, in general, $m$ need not be cosmooth. However, it is always possible to find a polar set $N$ such that $m$ is smooth relative to $\hat{X}$ restricted to $E-N$. This section is devoted to proving this statement. Of course, the deletion of a polar set from the state space is a trivial modification of the process $\hat{X}$.

Let us begin with an example which shows that a smooth measure need not be cosmooth, and which at the same time illustrates rather well the general situation. Let $E$ be the real line and let $X$ be the stable subordinator of index $\frac{1}{2}$; see [2]. Let the basic measure $\xi$ be Lebesgue measure; then probabilistically, starting at 0 , the dual process $\hat{X}$ is just $-X$. Moreover the potential kernel is given by

$$
U(x, y)=\left\{\begin{array}{lll}
\alpha(y-x)^{-1 / 2} & \text { if } & y>x, \\
0 & \text { if } & y \leqq x,
\end{array}\right.
$$

where $\alpha$ is a positive constant. Any finite open interval $G=(a, b)$ is an exit set 
and the corresponding state spaces are $B=[a, b), \hat{B}=(a, b]$ while the potential kernel $V$ is just $V(x, y)=U(x, y)$ for $x \in B, y \in \hat{B}$. Finally a basis for the fine topology is given by intervals of the form $(a, b)$ and $[a, b)$, while a basis for the cofine topology is given by intervals of the form $(a, b)$ and $(a, b], a<b$.

Let $g(x)=(-x)^{-1 / 2}$ if $x<0$ and $g(x)=1$ if $x \geqq 0$ and set $m(d x)=g(x) d x$. Clearly $m$ charges no semi-polar set and is strictly positive on both nonvoid finely open sets and nonvoid cofinely open sets. If $G=(a, b)$ with $a$ and $b$ finite, then it is immediate that $V m$ is bounded and so $m$ is smooth. The corresponding additive functional $A$ is given by $A(t)=\int_{0}^{t} g[X(s)] d s$. But any cofine neighborhood of 0 must contain a set of the form $(a, 0]$ and if $V$ is the potential kernel for $X$ terminated when it leaves $(a, 0]$, a simple calculation shows that $m V(0)=\infty$, and so $m$ cannot be cosmooth. However, if we delete the (polar) set $\{0\}$ from $E$ it is not difficult to see that $m$ is smooth relative $\hat{X}$ on $E-\{0\}$. That is, we only consider $\hat{X}$ starting from an $x \neq 0$. Again the corresponding additive functional is given by $A(t)=\int_{0}^{t} g[X(t)] d t$ which is $\hat{P}^{x}$ a.s. finite for all $t$ provided $x \neq 0$. It is easy to see that $A(t)=\infty$ for all $t>0$ with $\hat{P}^{0}$ probability one. Thus in this special case $m$ becomes cosmooth if we delete a polar set from the state space, and as we will see this is the typical situation in the general case.

THEOREM 8.1. Let $\mu$ be a measure on $E$ that charges no semi-polar set and is strictly positive on nonvoid finely open Borel sets. Then $\mu$ is strictly positive on nonvoid cofinely open Borel sets.

Proof. Let $D$ be a nonvoid cofinely open Borel set with compact closure and let $B$ denote the set of points which are not coregular for $D^{c}$. Then $B \supset D, B$ is a cofinely open Borel set, and $B-D$ is semi-polar so that $\mu(B-D)=0$. Now let $B_{1}$ denote the set of points which are not regular for $B^{c}$; then $B_{1}-B$ and $B-B_{1}$ are semi-polar by Theorem 2.1. Also $B_{1}$ is a finely open Borel set and $B_{1}$ is nonvoid, since if $B_{1}$ were empty then $B$ would be semi-polar which is impossible because $B$ is a nonvoid cofinely open set. Hence

$$
0<\mu\left(B_{1}\right)=\mu(B)=\mu(D) .
$$

This completes the proof of Theorem 8.1.

LEMMA 8.2. Let $G$ be an open set with compact closure and suppose $\bar{G} \subset H$ where $H$ is an exit set. Let $T=T_{H^{\prime}}$. If $D$ is polar relative to $(X, T)$ then $D \cap G$ is polar.

Proof. Let $K$ be open with $G \subset K, R \subset H$ and let $R=T_{K}$. Every point of $D \cap G$ is coregular for $K$ and so Proposition 18.5 of $[5, \mathrm{III}]$ implies that the point $X(R)$ is regular for $D \cap \bar{G}$ a.s. on the set $\{X(R) \in D \cap G, R<\infty\}$. However, no point is regular for $D \cap G$ since $D$ is polar relative to $(X, T)$ and therefore $P^{x}[X(R) \in D \cap G ; R<\infty]=0$ for all $x$. We now define $T_{1}=0$ and 


$$
\begin{aligned}
& S_{n}=T_{n}+T\left(\theta_{T_{n}}\right), \quad n \geqq 1, \\
& T_{n+1}=S_{n}+R\left(\theta_{S_{n}}\right), \quad n \geqq 1,
\end{aligned}
$$

so that $T_{1} \leqq S_{1}<T_{2}<S_{2}<\cdots$ whenever the quantities involved are finite. Clearly $P^{x}\left[S_{n} \rightarrow \infty\right]=1$ for all $x$. We have $X(t)$ not in $K$ a.s. on each interval $\left[S_{n}, T_{n+1}\right)$ and $X(t)$ not in $D \cap \bar{G}$ a.s. on each interval $\left(T_{n}, S_{n}\right)$ since $D$ is polar relative to $(X, T)$. But the third sentence of the proof implies that $P^{x}\left[X\left(T_{n}\right) \in D \cap \bar{G}\right.$ for some $n>1]=0$ for all $x$, and consequently $P^{x}[X(t) \in D \cap \bar{G}$ for some $t>0]=0$ for all $x$.

From now on let $m$ be a fixed smooth measure. We intend to show that by altering the state space trivially one can associate with $m$ an additive functional of $\hat{X}$ on the altered state space. We begin the construction locally.

THEOREM 8.3. Let $G$ and $H$ be exit sets with $\bar{G} \subset H$. Let $T=T_{H^{\prime}}$ and let $V, B$, and $K$ denote the potential kernel, the state space, and the hitting distributions for $(X, T)$. Then the following statements are valid:

(i) The sequence $\left\{B_{n}\right\}$ whose union is $B$ that appears in the definition of the smoothness of $m$ (relative to $H$ ) may be chosen so that $m\left(B_{n}\right)$ is finite for all $n$.

(ii) If $T_{n}=T_{B_{n}^{c}}$ where $\left\{B_{n}\right\}$ is the sequence in (i) and $S=T_{G^{\prime}}$ then $\hat{P}^{x}\left[T_{n} \geqq S\right] \rightarrow 1$ as $n \rightarrow \infty$ for almost all $x$.

(iii) Let $A$ denote the exceptional set in (ii) and $R=T_{G \cap A}$. Then $\hat{P}^{x}[R<S]=0$ for all $x$ in $\bar{G}-A$.

(iv) $G \cap A$ is polar relative to $(\hat{X}, S)$.

Proof. (i) Let $G^{*}$ be an exit set with $\bar{H} \subset G^{*}$. Let $V^{*}, B^{*}$ have their usual meaning relative to $G^{*}$. Since $m$ is smooth there exists a sequence $\left\{D_{n}\right\}$ of finely open Borel sets whose union is $B^{*}$ and such that (a) $\phi_{n}(x)=\int_{D_{n}} V^{*}(x, y) m(d y)$ is bounded and (b) $P^{x}\left[T_{D_{n}^{c}}=T^{*}\right] \rightarrow 1$ as $n \rightarrow \infty$. If $L$ is an open neighborhood of $\bar{H}$ with $L \subset G^{*}$ then Lemma 2.1 of [1] easily implies that $L$ is cospecial (and special) with respect to $\left(X, T^{*}\right)$. Therefore Proposition 12.1 of $[5$, II $]$ together with the boundedness of $\phi_{n}$ quickly yield the fact that $m\left(D_{n} \cap L\right)$ is finite. Define $B_{n}=D_{n} \cap B \subset D_{n} \cap L$. Clearly the sequence $\left\{B_{n}\right\}$ has the desired properties.

(ii) Since $P^{x}\left(T_{n}=T\right) \rightarrow 1$ it follows that $K_{n}(x, E) \rightarrow 0$ for all $x$ where $K_{n}=K_{B_{n}^{c}}$, and so

$$
\int d z \int V \hat{K}_{n}(x, z) d x \rightarrow 0 .
$$

But the inner integral decreases as $n$ increases and hence $\int V \hat{K}_{n}(x, z) d x \rightarrow 0$ as $n \rightarrow \infty$ for almost all $z$. However $G$ is contained in a cospecial set and therefore $\int V(x, y) d x$ is bounded away from zero on $\bar{G}$. (We use here as in (i) Lemma 
2.1 of [1] and Proposition 12.1 of [5, II].) Thus $\hat{K}_{n}(\bar{G}, x) \rightarrow 0$ a.e., which clearly yields $\hat{P}^{x}\left[T_{n} \geqq S\right] \rightarrow 1$ for almost all $x$.

(iii) Suppose (iii) does not hold; then there is a $z$ in $G-A$ and a compact set $D \subset G \cap A$ such that $\hat{P}^{z}\left[T_{D}<S\right]>0$. Indeed using (ii) we may pick a $k$ so large that

$$
\hat{P}^{z}\left[T_{D}<S \leqq T_{k}\right]>0
$$

Let $n>k$; then

$$
\begin{aligned}
\hat{P}^{z}\left(T_{n}<S\right) & \geqq \hat{P}^{z}\left[T_{n}<S, T_{D}<S, T_{D}<T_{n}\right] \\
& =\hat{E}^{z}\left\{\hat{P}^{X\left(T_{D}\right)}\left[T_{n}<S\right] ; T_{D}<S, T_{D}<T_{n}\right\} \\
& \geqq \hat{E}^{z}\left\{\hat{P}^{X\left(T_{D}\right)}\left[T_{n}<S\right] ; T_{D}<S \leqq T_{k}\right\} .
\end{aligned}
$$

But $\hat{P}^{z}\left(T_{n}<S\right) \rightarrow 0$ as $n \rightarrow \infty$ since $z$ is not in $A$, while $X\left(T_{D}\right) \in D \subset A$ a.s. on $\left\{T_{D}<S\right\}$ and so $\lim \hat{P}^{X\left(T_{D}\right)}\left[T_{n}<S\right]>0$ a.s. on $\left\{T_{D}<S\right\}$. (This limit exists since the $T_{n}$ are increasing.) We now have a contradiction and hence (iii) is established.

(iv) Let $x$ be a fixed point in $\bar{G}$ and choose a sequence $\left\{t_{j}\right\}$ decreasing to zero such that

$$
\hat{P}^{x}\left[X\left(t_{j}\right) \in \bar{G} \cap A ; t_{j}<S\right]=0
$$

for each $j$. This is possible since $\xi(A)=0$. Now

$$
\begin{aligned}
& \hat{P}^{x}\left[X(t) \in G \cap A \text { for some } t \in\left(t_{j}, S\right)\right] \\
& \quad \leqq \hat{E}^{x}\left\{\hat{P}^{X\left(t_{j}\right)}(R<S) ; t_{j}<S\right\},
\end{aligned}
$$

and (iii) and (8.1) imply that this last expression is zero. Letting $t_{j} \rightarrow 0$ we obtain

$$
\hat{P}^{x}[X(t) \in G \cap A \text { for some } t \in(0, S)]=0,
$$

which is the desired conclusion.

THEOREM 8.4. Let $G$ be an exit set and let $T, V, B$, etc., have their usual meanings relative to $G$. There exist a polar Borel set $N$ and a c.a.f. $A$ of $(\hat{X}, T)$ restricted to $\hat{B}-N$ such that for any $f \geqq 0$

$$
\hat{E}^{x} \int_{0}^{T} f[X(t)] d \hat{A}(t)=\int m(d y) f(y) V(y, x)
$$

for all $x$ not in $N$.

Proof. Note that since $N$ is polar restricting $(\hat{X}, T)$ to $\hat{B}-N$ means that we only look at the trajectories of $(\hat{X}, T)$ which start from points not in $N$. Also it is clear that $E-N$ is both finely and cofinely open. Now let $H$ be an exit set with $\bar{G} \subset H$ and let $W$ be the potential kernel for $\left(X, T_{H^{\prime}}\right)$. According to Theorem 
8.3 (i) we can choose the sequence $\left\{D_{n}\right\}$ that appears in the definition of the smoothness of $m$ (relative to the exit set $H$ ) so that $m\left(D_{n}\right)$ is finite. If $\mu_{n}$ is the restriction of $m$ to $B_{n}=B \cap D_{n}(B$ is the state space for $(X, T))$, then

$$
\int \mu_{n} W(x) d x \leqq \mu_{n}(E) \sup \left(\int W(y, x) d x\right)<\infty
$$

and so $\mu_{n} W$ is finite a.e. Therefore $\left\{\mu_{n} W=\infty\right\}$ is $\left(\hat{X}, T_{H^{\prime}}\right)$ polar and hence Lemma 8.2 implies that $N_{1}=\bar{G} \cap\left\{\mu_{n} W=\infty\right\}$ is polar. Also let $L$ be a neighborhood of $\bar{G}$ with $\bar{L} \subset H$; then Theorem 8.3 (iv) implies that $N_{2}=G \cap\left\{x: \hat{P}^{x}\left(T_{D_{n}^{c}} \geqq T_{L^{\prime}}\right) \nrightarrow 1\right\}$ is polar relative to $\left(X, T_{L^{\prime}}\right)$ and hence polar. We let $N$ be a polar Borel set containing $N_{1} \cup N_{2}$.

As above $B_{n}=B \cap D_{n}$ and $\mu_{n}$ is the restriction of $m$ to $B_{n}$ so that $\mu_{n}(E)<\infty$ and $\mu_{n} V \leqq \mu_{n} W$ is finite off $N$. Define $B_{n k}=\left\{\mu_{n} V \leqq k\right\} \cap \hat{B}$ and let $\mu_{n k}$ be the restriction of $\mu_{n}$ to $B_{n k}$. Since $\mu_{n}$ charges no semi-polar set it follows easily that $\mu_{n k} \leqq \mu_{n} K_{B_{n k}}$ and so $\mu_{n k} V \leqq k$ everywhere. Therefore the argument of the first part of the proof of Theorem 6.2 is applicable and consequently we obtain a c.a.f. $A_{n k}$ of $(\hat{X}, T)$ such that

$$
\hat{E}^{x} \int_{0}^{T} f[X(t)] d A_{n k}(t)=\int \mu_{n k}(d y) f(y) V(y, x)
$$

for all $f \geqq 0$ and all $x$. The usual compatibility argument shows that for fixed $n$ and $j \geqq k$ we have $A_{n k}=A_{n j}$ on $\left[0, T_{n k}\right]$ a.s. $\hat{P}^{x}$ for all $x$, where $T_{n k}$ is the time the process first leaves $B_{n k}$. But if $x$ is not in $N$ then

$$
\infty>\mu_{n} V(x) \geqq \mu_{n} V \hat{K}_{B_{n k}^{c}}^{c}(x) \geqq k \hat{P}^{x}\left[T_{n k}<T\right],
$$

and so $\hat{P}^{x}\left[T_{n k}<T\right] \rightarrow 0$ as $k \rightarrow \infty$ for each $x$ not in $N$. Thus we may define $A_{n}(t)=\lim _{k} A_{n k}(t)$ and obtain a c.a.f. of $(\hat{X}, T)$ restricted to $\hat{B}-N$ with $A_{n}(T)$ finite a.s. with respect to $\hat{P}^{x}$ for each $x$ not in $N$. Arguing as in the last paragraph of the proof of Theorem 6.2 yields

$$
E^{x} \int_{0}^{T} f\left(X_{t}\right) d A_{n}(t)=\int \mu_{n}(d y) f(y) V(y, x)
$$

for all $x$ not in $N$ and all $f \geqq 0$.

Once again we find that if $k \geqq n$ then $A_{k}=A_{n}$ on $\left[0, T_{n}\right]$ a.s. $\hat{P}^{x}$ for all $x$ not in $N$, where $T_{n}$ is the time the process first leaves $B_{n}$. Also it follows quickly from the definition of $N_{2}$ that $\hat{P}^{x}\left[T_{n}<T\right] \rightarrow 0$ if $x$ is not in $N$. Therefore we may define $\hat{A}(t)=\lim _{n} A_{n}(t)$ and obtain a c.a.f. of $(\hat{X}, T)$ restricted to $\hat{B}-N$ with $\hat{A}(T)$ finite a.s. relative to each $\hat{P}^{x}$ for $x$ not in $N$. One then establishes (8.2) as before. Finally observe that given the exceptional set $N$ then $\hat{A}$ is uniquely determined by (8.2), that is, if $\hat{C}$ is another c.a.f. of $(\hat{X}, T)$ restricted to $\hat{B}-N$ satisfying (8.2) then $\hat{A}=\hat{C}$ on $[0, T]$ with $\hat{P}^{x}$ probability one for each $x$ not in $N$.

The next theorem extends the local construction as in Theorem 6.3. 
THEOREM 8.5. There exist a polar Borel set $N$ and a c.a.f. $\hat{A}$ of $\hat{X}$ restrictdv to $\hat{E}=E-N$ such that for each exit set $G$ and $x$ not in $N$ we haee $\hat{P}^{x}[\hat{A}(T)<\infty]=1$ and

$$
\hat{E}^{x} \int_{0}^{T} f[X(t)] d \hat{A}(t)=\int m(d y) f(y) V(y, x)
$$

for each $f \geqq 0$. Here $T$ and $V$ have their usual meanings.

Proof. Let $\left\{G_{j}\right\}$ be a covering of $E$ by exit sets with the properties of Theorem 3.2. Let $N_{j}$ be the polar Borel set and $\hat{A}_{j}$ the c.a.f. relative to $G_{j}$ constructed in Theorem 8.4. Let $N=\bigcup N_{j}$ so that $N$ is polar. If $R_{n}$ has the same meaning as in the proof of Theorem 6.3 then it follows that $R_{n} \rightarrow \sigma$ a.s. $\hat{P}^{x}$ for all $x$. The additive functional $\hat{A}$ of $\hat{X}$ restricted to $\hat{E}=E-N$ can then be constructed just as in the proof of Theorem 6.3.

Just as before one can show that $\hat{A}$ will be strictly increasing on $[0, \sigma)$ a.s. $\hat{P}^{x}$ for all $x$ in $E$ if and only if $m$ is strictly positive on nonvoid cofinely open Borel sets which, in view of Theorem 8.1, is equivalent to $m$ being strictly positive on nonvoid finely open Borel sets.

9. Duality and hitting distributions. Let $X$ and $\hat{X}$ have their usual meanings and, as in $\S 7$, let $Y=\left(E,{ }^{*} P^{x}\right)$ be another Hunt process with the same hitting distributions as $X$. Let $m$ be the speed measure of $Y$ so that $m$ is a smooth measure strictly positive on nonvoid finely open Borel sets. The results of $\S 8$ imply the existence of a polar set $N$ and a continuous additive functional $\hat{A}$ of $\hat{X}$ restricted to $\hat{E}=E-N$ which is strictly increasing a.s. on $[0, \sigma)$ and which corresponds to $m$ in the manner described in $\S 8$. If $\hat{\tau}$ is the functional inverse to $\hat{A}$ we can form the process

$$
\hat{Y}(t)=\hat{X}[\hat{\tau}(t)]
$$

with state space $\hat{E}$. (Sometimes it will be more convenient to consider $\hat{Y}$ as a process on $E$ but only consider it starting from points in $E$.) It is easy to see that $\hat{Y}$ has all the important properties of a Hunt process and that $\hat{Y}$ and $\hat{X}$ have the same hitting distributions starting from any point in $\hat{E}$, since $N$ is polar. The problem then arises as to what extent the processes $Y$ and $\hat{Y}$ are in duality. The present section is devoted to investigating this question.

We will denote the expectation operators for $\hat{Y}$ by ${ }^{*} \hat{E}^{x}$ defined for $x \in \hat{E}=E-N$. Let $G$ denote a fixed exit set and let $V, T, B$, etc., have their usual meanings. As was seen in the proof of Theorem 8.4 we can choose a sequence $\left\{B_{n}\right\}$ of finely open Borel sets with the following properties:

(i) If $\mu_{n}$ is the restriction of $m$ to $B_{n}$ then $\mu_{n}(E)<\infty, V \mu_{n}$ is bounded, and $\mu_{n} V(x)<\infty$ if $x$ is not in $N$.

(ii) If $T_{n}=T_{B_{n}^{c}}$ then $P^{x}\left(T_{n}=T\right) \rightarrow 1$ for all $x$ and $\hat{P}^{x}\left[T_{n}=T\right] \rightarrow 1$ for all $x$ not in $N$. 
There is no loss of generality in assuming that the state space for $\left(X, T_{n}\right)$ is $B_{n}$. Let $\hat{B}_{n}$ be the state space for $\left(\hat{X}, T_{n}\right)$ and $B_{n}^{*}=\hat{B}_{n}-N$. For the time being let us suppress the index $n$ so that $B$ denotes a fixed $B_{n}, T=T_{n}, \mu$ is the restriction of $m$ to $B$, and $V$ is the potential kernel for $(X, T)$. Let us introduce the potential operators for $(Y, T)$ and $(\hat{Y}, T)$ defined by

$$
\begin{aligned}
& Q^{\lambda}(x, A)={ }^{*} E^{x} \int_{0}^{T} e^{-\lambda t} I_{A}[X(t)] d t, \quad x \in E, \\
& \hat{Q}^{\lambda}(A, x)=* \hat{E}^{x} \int_{0}^{T} e^{-\lambda t} I_{A}[X(t)] d t, \quad x \in \hat{E}=E-N,
\end{aligned}
$$

where $I_{A}$ is the indicator function of $A$. It follows from the discussion in $\S 7$ and the construction of $\hat{Y}$ that

$$
\begin{array}{ll}
Q^{0}(x, d y)=V(x, y) \mu(d y), & x \in E, \\
\hat{Q}^{0}(d y, x)=\mu(d y) V(y, x), & x \in \hat{E} .
\end{array}
$$

The next theorem is the basic step in setting up the duality between $Y$ and $\hat{Y}$.

THEOREM 9.1. For each $\lambda \geqq 0$ there exists a function $q^{\lambda}(x, y)$ defined on $E \times E$ with the following properties:

(i) $x \rightarrow q^{\lambda}(x, y)$ is $\lambda$-excessive relative to $(Y, T)$ for each $y \in \hat{E}$ and $y \rightarrow q^{\lambda}(x, y)$ is $\lambda$-excessive relative to $(\hat{Y}, T)$ for each $x \in E$.

(ii) $Q^{\lambda}(x, d y)=q^{\lambda}(x, y) \mu(d y)$ for $x \in E$ and $\hat{Q}^{\lambda}(d y, x)=\mu(d y) q^{\lambda}(y, x)$ for $x \in \hat{E}$.

(iii) $q^{\lambda}(x, y)=V(x, y)-\lambda \int Q^{\lambda}(x, d z) V(z, y)=V(x, y)-\lambda \int V(x, z) \hat{Q}^{\lambda}(d z, y)$ for all $(x, y)$ in $(E \times \hat{E})-\Gamma$ where $\Gamma=\{(x, y): V(x, y)=\infty\}$.

Proof. Since $z \rightarrow V(z, y)$ is $(X, T)$ and hence $(Y, T)$ excessive for each $y \in E$ it follows that $\lambda Q^{\lambda} V \leqq V$ everywhere and so we may define

$$
q^{\lambda}(x, y)=V(x, y)-\lambda \int Q^{\lambda}(x, d z) V(z, y)
$$

for $(x, y)$ not in $\Gamma$. Then $q^{\lambda} \geqq 0$ for such $(x, y)$. We leave $q^{\lambda}$ undefined on $\Gamma$ for the moment. Of course $V$, and hence $q^{\lambda}$, vanish off $B \times \hat{B}$. Also it is immediate from the resolvent equation that $Q^{\lambda}(x, d y)=q^{\lambda}(x, y) \mu(d y)$ for all $x$ in $E$. This last expression makes sense since $\Gamma_{x}=\{y: V(x, y)=\infty\}$ is polar relative to $(\hat{X}, T)$ and hence certainly $\mu$ null. Also $\Gamma^{y}=\{x: V(x, y)=\infty\}$ is polar relative to $(X, T)$.

Let $y \in E$ be fixed; then for $\eta>0$ and $x$ not in $\Gamma^{y}$ we have

$$
\eta Q^{\lambda+\eta} q^{\lambda}(x, y)=\eta Q^{\lambda+\eta} V(x, y)-\lambda \eta Q^{\lambda+\eta} Q^{\lambda} V(x, y),
$$

and both terms on the right are finite since $x$ is not in $\Gamma^{y}$. But $\eta Q^{\lambda+\eta} Q^{\lambda}=Q^{\lambda}-Q^{\lambda+\eta}$ and so

$$
\eta Q^{\lambda+\eta} q^{\lambda}(x, y)=(\eta+\lambda) Q^{\lambda+\eta} V(x, y)-\lambda Q^{\lambda} V(x, y) .
$$


Since $x \rightarrow V(x, y)$ is $\lambda$-excessive relative to $(Y, T)$ the first term on the right increases to $V(x, y)$ as $\eta \rightarrow \infty$. Thus we have shown

$$
\eta Q^{\lambda+\eta} q^{\lambda}(x, y) \uparrow q^{\lambda}(x, y)
$$

as $\eta \rightarrow \infty$ provided $(x, y)$ is not in $\Gamma$. Now define $g^{\lambda}=q^{\lambda}$ off $\Gamma$ and $g^{\lambda}=\infty$ on $\Gamma$. It is immediate from (9.3) that $\eta Q^{\lambda+\eta} g^{\lambda} \leqq g^{\lambda}$, and so for each fixed $y$ the function $x \rightarrow \eta Q^{\lambda+\eta} g^{\lambda}(x, y)$ increases to a function $\lambda$-excessive relative to $(Y, T)$ as $\eta \rightarrow \infty$. Moreover it is clear from (9.3) that this limit agrees with $q^{\lambda}(x, y)$ for $x$ not in $\Gamma^{y}$. Thus if we set

$$
q^{\lambda}=\lim _{\eta \rightarrow \infty} \eta Q^{\lambda+\eta} g^{\lambda}=\lim _{\eta \rightarrow \infty} \eta Q^{\lambda+\eta} q^{\lambda}
$$

everywhere, then this agrees with our previous definition (9.2) off $\Gamma$ and $x \rightarrow q^{\lambda}(x, y)$ is $\lambda$-excessive with respect to $(Y, T)$ for all $y$.

Now define $R^{\lambda}(d y, x)=\mu(d y) q^{\lambda}(y, x)$. We want to show that $R^{\lambda}(\cdot, x)=\hat{Q}^{\lambda}(\cdot, x)$ for all $x$ not in $N$, and we already know that this is true when $\lambda=0$. Let $L_{1}=L_{1}(\mu)$. Since $V \mu$ is bounded (say by $M$ ) and since $q^{\lambda}(x, y) \leqq V(x, y)$ we see that $R^{\lambda}: L_{1} \rightarrow L_{1}$ and that $\left\|R^{\lambda} f\right\|_{1} \leqq M\|f\|_{1}$ for all $\lambda \geqq 0$. Also $\hat{Q}^{\lambda}(d y, x) \leqq \hat{Q}^{0}(d y, x)=\mu(d y) V(y, x)$ for $x$ not in $N$ and so $\hat{Q}^{\lambda}: L_{1} \rightarrow L_{1}$ and $\left\|\hat{Q}^{\lambda} f\right\|_{1} \leqq M\|f\|_{1}$ for all $\lambda \geqq 0$, since $N$ is $\mu$ null.

Since $\left\{Q^{\lambda} ; \lambda \geqq 0\right\}$ satisfies the resolvent equation on the space of bounded measurable functions we have for $\lambda>\eta \geqq 0$

$$
q^{\lambda}(x, y)-q^{\eta}(x, y)=(\eta-\lambda) \int q^{\lambda}(x, z) q^{\eta}(z, y) \mu(d z)
$$

a.e. $(\mu)$ in $y$ for each fixed $x$. The Fubini theorem then implies that $\left\{R^{\lambda} ; \lambda \geqq 0\right\}$ satisfies the resolvent equation on $L_{1}$.

Let $M_{1}=R^{0} L_{1}=\hat{Q}^{0} L_{1}$ and $M_{2}=R^{0} M_{1}=\hat{Q}^{0} M_{1}$. Using the fact that $\left\|R^{\lambda}\right\|_{1}$ and $\left\|\hat{Q}^{\lambda}\right\|_{1}$ are bounded in $\lambda$ on $\lambda \geqq 0$, standard manipulations with the resolvent equation yield the result that $R^{\lambda}$ and $\hat{Q}^{\lambda}$ are one-to-one on $M_{2}$. Since $R^{0}=\hat{Q}^{0}$ it is again a standard argument to conclude that $R^{\lambda}=\hat{Q}^{\lambda}$ on $M_{2}$ for all $\lambda \geqq 0$. If $f$ is continuous with compact support (and hence in $L_{1}$ since $\mu$ is a finite measure) it follows from the right continuity of the paths of $\hat{Y}$ that $\alpha \hat{Q}^{\alpha} f(x) \rightarrow f(x)$ as $\alpha \rightarrow \infty$ for all $x$ in $\hat{B}-N$, hence in $L_{1}$ since $\mu$ is concentrated on $B \cap \hat{B}$ and $\left|\alpha \hat{Q}^{\alpha} f\right| \leqq \sup |f|$. Also $\beta \hat{Q}^{\beta} \hat{Q}^{\alpha} f \rightarrow \hat{Q}^{\alpha} f$ as $\beta \rightarrow \infty$ pointwise boundedly and hence in $L_{1}$ since $\left\|\hat{Q}^{\beta}\right\|_{\infty} \leqq \beta^{-1}$. Therefore

$$
R^{\lambda}\left[\alpha \beta \hat{Q}^{\beta} \hat{Q}^{\alpha} f\right]=\hat{Q}^{\lambda}\left[\alpha \beta \hat{Q}^{\beta} \hat{Q}^{\alpha} f\right],
$$

and so letting $\beta \rightarrow \infty$ and then $\alpha \rightarrow \infty$ we obtain $R^{\lambda} f=\hat{Q}^{\lambda} f$ as elements of $L_{1}$. However, if $f \geqq 0$ then $\hat{Q}^{\lambda} f$ is $\lambda$-excessive with respect to $(\hat{Y}, T)$ and it is easy to see that this implies that $\hat{Q}^{\lambda} f$ is cofinely continuous off $N$. Also $R^{\lambda} f$ is the difference of two $(\hat{X}, T)$ excessive functions and hence is cofinely continuous 
where both are finite. But $\mu V<\infty$ off $N$ and this yields the fact that $R^{\lambda} f$ is cofinely continuous off $N$. Therefore $R^{\lambda} f(x)=\hat{Q}^{\lambda} f(x)$ for all $x$ not in $N$ and all continuous $f \geqq 0$ with compact support, and so we finally obtain $\hat{Q}^{\lambda}(d y, x)=\mu(d y) q^{\lambda}(y, x)$ for all $x$ not in $N$.

So far $q^{\lambda}(x, y)$ is defined on $E \times E$ and $x \rightarrow q^{\lambda}(x, y)$ is $\lambda$-excessive with respect to $(Y, T)$ for each fixed $y$. We must still investigate the function $y \rightarrow q^{\lambda}(x, y)$. We begin by defining for $y$ not in $N$ and $x$ not in $\Gamma^{y}$ the function

$$
\hat{q}^{\lambda}(x, y)=V(x, y)-\lambda \int V(x, z) \hat{Q}^{\lambda}(d z, y)
$$

which is easily seen to be well defined and non-negative for $(x, y)$ in $(E \times \hat{E})-\Gamma$. We extend $\hat{q}^{\lambda}$ to $E \times \hat{E}$ in a manner analogous to that used in the extension of $q^{\lambda}$ from $(E \times E)-\Gamma$ to $E \times E$. This extension is such that $y \rightarrow \hat{q}^{\lambda}(x, y)$ is $\lambda$-excessive with respect to $(\widehat{Y}, T)$ for each fixed $x$. Clearly

$$
\mu(d x) \hat{q}^{\lambda}(x, y)=\hat{Q}^{\lambda}(d x, y), \quad y \notin N .
$$

But this implies that $q^{\lambda}=\hat{q}^{\lambda}$ on $(E \times \hat{E})-\Gamma$. Recalling the method used in the extension of $q^{\lambda}$ and $\hat{q}^{\lambda}$ we have for $y$ not in $N$

$$
\begin{aligned}
q^{\lambda}(x, y) & =\lim _{\eta \rightarrow \infty} \eta Q^{\lambda+\eta} q^{\lambda}(x, y) \\
& =\lim _{\eta \rightarrow \infty} \eta \int q^{\lambda+\eta}(x, z) \mu(d z) q^{\lambda}(z, y) \\
& =\lim _{\eta \rightarrow \infty} \eta \int \hat{q}^{\lambda+\eta}(x, z) \mu(d z) \hat{q}^{\lambda}(z, y) \\
& =\hat{q}^{\lambda}(x, y) .
\end{aligned}
$$

Thus $q^{\lambda}=\hat{q}^{\lambda}$ on $E \times \hat{E}$ and this completes the proof of Theorem 9.1.

A consequence of Theorem 9.1 is that

$$
\int V(x, z) q^{\lambda}(z, y) \mu(d z)=\int q^{\lambda}(x, z) V(z, y) \mu(d z)
$$

first on $E-\Gamma^{y}$ for each fixed $y$ not in $N$ and then on $E \times \hat{E}$ since both sides of the above equation are finely continuous in $x$ for each fixed $y$ not in $N$.

Recalling the discussion preceding Theorem 9.1 we reintroduce the index $n$; thus for our (still) fixed exit set $G$ we have $B=\bigcup B_{n}$ where the $B_{n}$ have the properties listed above Theorem 9.1. If we let $T_{n}=T_{B_{n}^{c}}$ and $V_{n}$ be the potential kernel for $\left(X, T_{n}\right)$ we can apply Theorem 9.1 for each $n$ and obtain using an obvious notation

$$
\begin{array}{ll}
Q_{n}^{\lambda}(x, d y)=q_{n}^{\lambda}(x, y) m(d y) & \text { for all } x, \\
\hat{Q}_{n}^{\lambda}(d y, x)=m(d y) q_{n}^{\lambda}(y, x), & x \notin N .
\end{array}
$$


If $f \geqq 0$ then $Q_{n}^{\lambda} f(x) \leqq Q_{k}^{\lambda} f(x)$ for each fixed $x$ and $k>n$. Therefore $q_{n}^{\lambda}(x, \cdot) \leqq q_{k}^{\lambda}(x, \cdot)$, a.e. $(m)$, on $\hat{E}=E-N$, and hence everywhere since the functions in question are cofinely continuous on $\hat{E}$. Thus we may define

$$
q^{\lambda}(x, y)=\lim _{n \rightarrow \infty} q_{n}^{\lambda}(x, y)
$$

on $E \times E$, and the limit in question is increasing with $n$. But $P^{x}\left(T_{n}=T\right) \rightarrow 1$ for all $x$ and $\hat{P}^{x}\left(T_{n}=T\right) \rightarrow 1$ for all $x$ not in $N$, and consequently

$$
\begin{array}{ll}
Q^{\lambda}(x, d y)=q^{\lambda}(x, y) m(d y), & x \in E, \\
\hat{Q}^{\lambda}(d y, x)=m(d y) q^{\lambda}(y, x), & x \in \hat{E},
\end{array}
$$

where $q^{\lambda}$ is as defined in (9.7).

Fix $y$ in $\hat{E}=E-N$ and fix $n$; if $k>n$ define

$$
f_{k}^{\lambda}(x)=I_{B_{n}}(x) q_{k}^{\lambda}(x, y) .
$$

Since $x \rightarrow q_{k}^{\lambda}(x, y)$ is $\lambda$-excessive relative to $\left(Y, T_{k}\right)$ it is easy to check that $f_{k}^{\lambda}$ is $\lambda$-excessive relative to $\left(Y, T_{n}\right)$ for all $k>n$. Moreover $f_{k}^{\lambda}(x)$ increases to $I_{B_{n}}(x) q^{\lambda}(x, y)=g_{n}^{\lambda}(x, y)$ as $k \rightarrow \infty$ and so $g_{n}^{\lambda}$ is $\lambda$-excessive relative to $\left(Y, T_{n}\right)$ and increases to $q^{\lambda}$ with $n$. Since

$$
e^{-\lambda t_{*}} E^{x}\left\{g_{n}^{\lambda}\left(X_{t}, y\right) ; t<T_{n}\right\} \leqq g_{n}^{\lambda}(x, y) \leqq q^{\lambda}(x, y),
$$

we obtain on letting $n \rightarrow \infty$

$$
e^{-\lambda t * E^{x}}\left\{q^{\lambda}\left(X_{t}, y\right) ; t<T\right\} \leqq q^{\lambda}(x, y)
$$

for each $x$ in $E$. Also

$$
\begin{aligned}
\lim _{t \rightarrow 0} & \inf e^{-\lambda t *} E^{x}\left\{q^{\lambda}\left(X_{t}, y\right) ; t<T\right\} \\
& \geqq \lim _{t \rightarrow 0} e^{-\lambda t *} E^{x}\left\{g_{n}^{\lambda}\left(X_{t}, y\right) ; t<T_{n}\right\} \\
& =g_{n}^{\lambda}(x, y) \rightarrow q^{\lambda}(x, y) \text { as } n \rightarrow \infty,
\end{aligned}
$$

and combining this with the previous inequality it follows that $x \rightarrow q^{2}(x, y)$ is $\lambda$-excessive relative to $(Y, T)$ for each $y$ in $\hat{E}$. Similarly $y \rightarrow q^{\lambda}(x, y)$ is $\lambda$-excessive relative to $(\hat{Y}, T)$ for each $x$ in $E$. We may now state the following theorem (still relative to our fixed exit set $G$ ).

THEOREM 9.2. For each $\lambda \geqq 0$ there exists a function $q^{\lambda}(x, y)$ defined on $E \times E$ such that $x \rightarrow q^{\lambda}(x, y)$ is $\lambda$-excessive relative to $(Y, T)$ and $y \rightarrow q^{\lambda}(x, y)$ is $\lambda$-excessive relative to $(\hat{Y}, T)$. Moreover

(i) $Q^{\lambda}(x, d y)=q^{\lambda}(x, y) m(d y), x \in E$,

(ii) $\hat{Q}^{\lambda}(d y, x)=m(d y) q^{\lambda}(y, x), x \in \hat{E}$,

(iii) $K_{D}^{\lambda} q^{\lambda}(x, y)=q^{\lambda} \hat{K}_{D}^{\lambda}(x, y)$ for all $(x, y)$ in $E \times \hat{E}$ and all Borel sets $D$. 
Proof. Everything except (iii) has already been proved above. However (iii) can now be proved in a manner similar to that used by Meyer in [10, Chapter 10]. We refer the reader to [10] for details. Of course $q^{0}(x, y)=V(x, y)$ and $q^{\lambda}(x, y)$ vanishes off $B \times(\hat{B}-N)$.

Theorem 9.2 establishes the duality between $(Y, T)$ and $(\hat{Y}, T)$ with respect to the speed measure $m$ of $Y$. In order to extend this duality to the nonterminated processes $Y$ and $\hat{Y}$ we first establish the duality in a special case.

THEOREM 9.3. Suppose that $E$ is the union of an increasing sequence $\left\{G_{j}\right\}$ of exit sets. Let $R^{\lambda}$ and $\hat{R}^{\lambda}$ denote the potential kernels $(\lambda>0)$ for $Y$ and $\hat{Y}$ respectively. Then there exists a function $r^{\lambda}$ defined on $E \times \hat{E}$ such that $R^{\lambda}(x, d y)=r^{\lambda}(x, y) m(d y)$ and $\hat{R}^{\lambda}(d y, x)=m(d y) r^{\lambda}(y, x)$. Moreover $x \rightarrow r^{\lambda}(x, y)$ is $\lambda$-excessive relative to $Y$ for each $y$ in $\hat{E}$ and $y \rightarrow r^{\lambda}(x, y)$ is $\lambda$-excessive relative to $\hat{X}$ for each $x$ in $E$.

Proof. Theorem 9.2 may be applied to each exit set $G_{j}$ in the increasing sequence $\left\{G_{j}\right\}$. We denote the corresponding function by $q_{j}^{\dot{\lambda}}(x, y)$. Now using an argument similar to the one given above Theorem 9.2 , one sees that $q_{j}^{\lambda}(x, y)$ increases on $E \times \hat{E}$ as $j \rightarrow \infty$ and that $r^{\lambda}(x, y)=\lim _{j \rightarrow \infty} q_{j}^{\lambda}(x, y)$ has the desired properties. Thus Theorem 9.3 is established.

We now state the main result of this section.

THEOREM 9.4. The conclusion of Theorem 9.3 is true without the assumpion that $E$ is the union of an increasing sequence of exit sets. Moreover for any analytic set $D$ one has

$$
P_{D}^{\lambda} r^{\lambda}(x, y)=r^{\lambda} \hat{P}_{D}^{\lambda}(x, y)
$$

for all $(x, y)$ in $E \times \hat{E}$ and $\lambda>0$.

Proof. Let $S$ be an exponentially distributed random variable with parameter one that is independent of $X$ and $\hat{X}$. Let $S^{\mu}=S / \mu$ for any $\mu>0$ so that $S^{\mu}$ is exponentially distributed with parameter $\mu$. Let $X^{\mu}$ and $\hat{X}^{\mu}$ be the processes $X$ and $\hat{X}$ terminated at time $S^{\mu}$, that is, $X^{\mu}(t)=X(t)$ if $t<S^{\mu}$ and $X^{\mu}(t)=\Delta$ if $t \geqq S^{\mu}$, and similarly for $\hat{X}^{\mu}$. Clearly $X^{\mu}$ and $\hat{X}^{\mu}$ are Hunt processes satisfying hypothesis (F) with respect to the same basic measure $\xi$ and for which the state space $E$ is an increasing union of exit sets (relative to $X^{\mu}$ and $\hat{X}^{\mu}$ ). The lifetime is now $\sigma^{\mu}=\sigma \wedge S^{\mu}$. We use the notation $a \wedge b$ for $\min (a, b)$. Of course the representation of $X^{\mu}$ and $\hat{X}^{\mu}$ as $\left(X, S^{\mu}\right)$ and $\left(\hat{X}, S^{\mu}\right)$ is not the canonical representation of these processes described in $\S 2$, but that causes no difficulties in what follows.

As above $m$ denotes the speed measure of $Y$ and $N$ the exceptional set such that $m$ is cosmooth on $\hat{E}=E-N$. Let $A$ and $\hat{A}$ be the strictly increasing additive functionals of $X$ and $\hat{X}$ (restricted to $\hat{E}$ ) corresponding to $m$. Define 


$$
A_{\mu}(t)=\left\{\begin{array}{ll}
A(t) & \text { if } t<S^{\mu}, \\
A\left(S^{\mu}\right) & \text { if } t \geqq S^{\mu} ;
\end{array} \quad \hat{A}_{\mu}(t)= \begin{cases}\hat{A}(t) & \text { if } t<S^{\mu}, \\
\hat{A}\left(S^{\mu}\right) & \text { if } t \geqq S^{\mu} .\end{cases}\right.
$$

Then $A_{\mu}$ is a continuous additive functional of $X^{\mu}$ that is a.s. continuous and strictly increasing on $\left[0, \sigma^{\mu}\right)$, and a similar statement holds for $\hat{A}_{\mu}$ relative to $\hat{X}^{\mu}$ restricted to $\hat{E}$. We now claim that $A_{\mu}$ is smooth relative to $X^{\mu}$. Since any open set with compact closure is an exit set for the pair $\left(X^{\mu}, X^{\mu}\right)$, we must show that $A_{\mu}\left(T_{K^{c}} \wedge S^{\mu}\right)$ is a.s. finite for all compact subsets $K$ of $E$. $\left(T_{A}\right.$ will always denote the first hit of $A$ by $X$ (or $\hat{X}$ ) so that the first hit of $K^{c}$ by $X^{\mu}$ is $T_{K^{c}} \wedge S^{\mu}$.) Since $A(t)$ is finite a.s. on $[0, \sigma)$ it is immediate that $A_{\mu}\left(T_{\mathrm{K}^{\mathrm{d}}} \wedge S^{\mu}\right)$ is a.s. finite on the set $\left\{T_{K^{c}} \wedge S^{\mu}<\sigma\right\}$. However on the complementary set we have $T_{K^{c}}=\sigma \leqq S^{\mu}<\infty$ and one can prove that $A(\sigma)$ is a.s. finite on the set $\left\{T_{K^{c}}=\sigma<\infty\right\}$ by the same argument as that used in the proof of Theorem 5.3. Thus $A_{\mu}$ is smooth relative to $X^{\mu}$.

Let $G$ be an exit set for $(X, \hat{X})$ and let $f \geqq 0$ be such that

$$
\int V(x, y) f(y) m(d y)=E^{x} \int_{0}^{T} f\left(X_{t}\right) d A(t)
$$

is bounded in $x$. Here $V, T$ have their usual meanings relative to $G$. Now the above expression equals

$$
\begin{aligned}
E^{x} \int_{0}^{T \wedge} s^{\mu} f\left(X_{t}\right) d A(t) & +E^{x}\left\{\int_{s^{\mu}}^{T} f\left(X_{t}\right) d A(t) ; S^{\mu}<T\right\} \\
& =E^{x} \int_{0}^{T \wedge s^{\mu}} f\left(X_{t}^{\mu}\right) d A_{\mu}(t)+\mu V^{\mu} V(f m)(x) .
\end{aligned}
$$

Hence using the resolvent equation

$$
\begin{aligned}
E^{x} \int_{0}^{T \wedge s^{\mu}} f\left(X_{t}^{\mu}\right) d A_{\mu}(t) & =\left(V-\mu V^{\mu} V\right)(f m)(x) \\
& =\int V^{\mu}(x, y) f(y) m(d y) .
\end{aligned}
$$

Of course $V^{\mu}$ is the potential kernel with parameter $\mu$ for $(X, \hat{X})$, or equivalently the potential kernel with parameter 0 for $\left(X^{\mu}, X^{\mu}\right)$. Since exit sets for $(X, X)$ cover $E$, it is immediate from (9.8) that the measure corresponding to $A_{\mu}$ is $m$. A similar calculation shows that $\hat{A}_{\mu}$ is the additive functional of $\hat{X}^{\mu}$ restricted to $\hat{E}$ corresponding to $m$ (the exceptional set is the fixed set $N$ ). Clearly $X^{\mu}\left(\hat{X}^{\mu}\right)$ induce the same fine (cofine) topology on $E$ as $X(\hat{X})$.

We now define $\tau_{\mu}$ and $\hat{\tau}_{\mu}$ as the functionals inverse to $A_{\mu}$ and $\hat{A}_{\mu}$, that is,

$$
\begin{aligned}
& \tau_{\mu}(t)=s \quad \text { if } t<A_{\mu}\left(\sigma \wedge S^{\mu}\right) \text { and } t=A_{\mu}(s), \\
& \tau_{\mu}(t)=\sigma \wedge S^{\mu} \quad \text { if } t \geqq A_{\mu}\left(\sigma \wedge S^{\mu}\right),
\end{aligned}
$$


with a similar definition of $\hat{\tau}_{\mu}$. If we recall the definition of $A_{\mu}$ and $\hat{A}_{\mu}$ it is immediate that

$$
\begin{array}{ll}
\tau_{\mu}(t)=\tau(t) & \text { if } t<A\left(\sigma \wedge S^{\mu}\right), \\
\tau_{\mu}(t)=\sigma \wedge S^{\mu} & \text { if } t \geqq A\left(\sigma \wedge S^{\mu}\right),
\end{array}
$$

with a similar relationship between $\hat{\tau}_{\mu}$ and $\hat{\tau}$. We next define

$$
Y^{\mu}(t)=X^{\mu}\left[\tau_{\mu}(t)\right] ; \hat{Y}^{\mu}(t)=\hat{X}^{\mu}\left[\hat{\tau}_{\mu}(t)\right],
$$

with state spaces $E$ and $\hat{E}$ respectively. Theorem 9.3 is now applicable, and so for each $\lambda>0$ there exists a function $r_{\mu}^{\lambda}(x, y)$ defined on $E \times E$ such that $x \rightarrow r_{\mu}^{\lambda}(x, y)$ is $\lambda$-excessive relative to $Y^{\mu}$ for each $y$ in $E$ and $y \rightarrow r_{\mu}^{\lambda}(x, y)$ is $\lambda$-excessive for $\hat{Y}^{\mu}$ for each $x$ in $E$. Moreover for any bounded $f \geqq 0$ we have

$$
\int r_{\mu}^{\lambda}(x, y) f(y) m(d y)=E^{x} \int_{0}^{\infty} e^{-\lambda t} f\left[X^{\mu}\left(\tau_{\mu}(t)\right)\right] d t
$$

for each $x$ in $E$, and also for each $y$ in $\hat{E}$

$$
\int m(d x) f(x) r_{\mu}^{\lambda}(x, y)=E^{y} \int_{0}^{\infty} e^{-\lambda t} f\left[X^{\mu}\left(\hat{\tau}_{\mu}(t)\right)\right] d t
$$

Let $x$ be fixed; then using (9.9)

$$
\begin{aligned}
E^{x} \int_{0}^{\infty} e^{-\lambda t} f\left[X^{\mu}\left(\tau_{\mu}(t)\right)\right] d t & =E^{x} \int_{0}^{\sigma \wedge S^{\mu}} e^{-\lambda A_{\mu}(t)} f\left(X^{\mu}(t)\right) d A_{\mu}(t) \\
& =E^{x} \int_{0}^{S / \mu} e^{-\lambda A(t)} f\left(X_{t}\right) d A(t) .
\end{aligned}
$$

Combining this with $(9.10)$ it follows that $r_{\mu}^{\lambda}(x, \cdot)$ increases a.e. $(m)$ as $\mu$ decreases. But $y \rightarrow r_{\mu}^{\lambda}(x, y)$ is cofinely continuous and so $r_{\mu}^{\lambda}(x, y)$ increases for each fixed $(x, y)$ in $E \times E$ as $\mu$ decreases. Define $r^{\lambda}(x, y)=\lim _{\mu \rightarrow 0} r_{\mu}^{\lambda}(x, y)$. It is then clear that

$$
\begin{aligned}
\int r^{\lambda}(x, y) f(y) m(d y) & =E^{x} \int_{0}^{\infty} e^{-\lambda A(t)} f\left(X_{t}\right) d A(t) \\
& ={ }^{*} E^{x} \int_{0}^{\infty} e^{-i t} f\left(X_{t}\right) d t \\
& =R^{\lambda} f(x),
\end{aligned}
$$

for each $x$ and $f \geqq 0$ where $R^{\lambda}(x, d y)$ is the potential kernel with parameter $\lambda$ Jor $Y$. A similar argument yields $\hat{R}^{\lambda}(d x, y)=m(d x) r^{\lambda}(x, y)$ for all $y$ in $\hat{E}$ where $\hat{R}^{\lambda}$ is the potential kernel with parameter $\lambda$ for $\hat{Y}$.

We must still investigate the functions $x \rightarrow r^{\lambda}(x, y)$ and $y \rightarrow r^{2}(x, y)$. Using the properties of $r_{\mu}^{\lambda}$ we have 


$$
\begin{aligned}
r_{\mu}^{\lambda}(x, y) & \geqq e^{-\lambda t} E^{x}\left[r_{\mu}^{\lambda}\left(Y_{\tau}^{\mu}, y\right)\right] \\
& =e^{-\lambda t} E^{x}\left\{r_{\mu}^{\lambda}\left[X^{\mu}\left(\tau_{\mu}(t)\right), y\right] ; t<A\left(\sigma \wedge S^{\mu}\right)\right\} \\
& =e^{-\lambda t} E^{x}\left\{r_{\mu}^{\lambda}[X(\tau(t)), y] ; t<A\left(\sigma \wedge S^{\mu}\right)\right\} .
\end{aligned}
$$

But as $\mu_{\downarrow} 0, A\left(\sigma \wedge S^{\mu}\right) \uparrow A(\sigma)$ and hence

$$
\begin{aligned}
r^{\lambda}(x, y) & \geqq e^{-\lambda t} E^{x}\left\{r^{\lambda}[X(\tau(t)), y] ; t<A(\sigma)\right\} \\
& =e^{-\lambda t *} E^{x}\left[r^{\lambda}\left(X_{t}, y\right)\right]
\end{aligned}
$$

for each $t$. On the other hand for each $\mu>0$

$$
\begin{aligned}
& \underset{t \rightarrow 0}{\liminf } e^{-\lambda t} E^{x}\left\{r^{\lambda}[X(\tau(t)), y] ; t<A(\sigma)\right\} \\
& \geqq \liminf _{t \rightarrow 0} e^{-\lambda t} E^{x}\left[r_{\mu}^{\lambda}\left(Y_{t}^{\mu}, y\right) ; t<A\left(\sigma \wedge S^{\mu}\right)\right] \\
&=r_{\mu}^{\lambda}(x, y),
\end{aligned}
$$

and letting $\mu \rightarrow 0$ we see that the first expression in the above display is not less than $r^{\lambda}(x, y)$. Combining this with the previous inequality one concludes that $x \rightarrow r^{\lambda}(x, y)$ is $\lambda$-excessive with respect to $Y$ for each $y$ in $\hat{E}$. In a similar manner one finds that $y \rightarrow r^{\lambda}(x, y)$ is $\lambda$-excessive with respect to $\hat{Y}$ for each $x$ in $E$.

Thus the first sentence in Theorem 9.4 is established. The second sentence can now be proved in a manner similar to that used by Meyer in [10, Chapter 10]. Once again, we omit the details.

\section{REFERENCES}

1. R. M. Blumenthal, An extended Markov property, Trans. Amer. Math. Soc. 85 (1957), 52-72.

2. R. M. Blumenthal and R. K. Getoor, Sample functions of stochastic processes with stationary independent increments, J. Math. Mech. 10 (1961), 493-516.

3. R. M. Blumenthal, R. K. Getoor, and H. P. McKean, Jr., Markov processes with identical hitting distributions, Illinois J. Math. 6 (1962), 402-420.

4. E. B. Dynkin, Theory of Markov processes, Prentice-Hall, Englewood Cliffs, N. J., 1961. (English transl.)

5. G. A. Hunt, Markov processes and potentials. I, II, and III, Illinois J. Math. 1 (1957), 44-93, 316-369; 2 (1958), 151-213.

6. M. Loève, Probability theory, 2nd ed., Van Nostrand, Princeton, N.J., 1960.

7. H. P. McKean, Jr. and H. Tanaka, Additive functionals of the Brownian path, Mem. Coll. Sci. Univ. Kyoto Ser. A Math. 33 (1961), 479-506.

8. P. A. Meyer, Fonctionelles multiplicatives et additives de Markov, Ann. Inst. Fourier (Grenoble) 12 (1962), 125-230.

9. M. G. Sur, Continuous additive functionals of Markov processes and excessive functions, Dokl. Akad. Nauk. SSSR 137 (1961), 800-803 = Soviet Math. Dokl. 2 (1961), 365-368.

10. Séminaire de théorie du potentiel (Séminaire Brelot-Choquet-Deny), Inst. H. Poincaré, Paris, Fifth year, 1960-1961.

UNIVERSITY OF WASHINGTON,

Seattle, Washington 